\title{
Review of blue perovskite light emitting diodes with optimization strategies for perovskite film and device structure
}

\author{
Zongtao $\mathrm{Li}^{1,2 *}$, Kai $\mathrm{CaO}^{1}$, Jiasheng $\mathrm{Li}^{1,2 *}$, Yong Tang${ }^{1}$, Xinrui Ding ${ }^{1}$ and \\ Binhai $\mathrm{Yu}^{1}$
}

Perovskite light emitting diodes (PeLEDs) have attracted considerable research attention because of their external quantum efficiency (EQE) of $>20 \%$ and have potential scope for further improvement. However, compared to red and green PeLEDs, blue PeLEDs have not been extensively investigated, which limits their commercial applications in the fields of luminance and full-color displays. In this review, blue-PeLED-related research is categorized by the composition of perovskite. The main challenges and corresponding optimization strategies for perovskite films are summarized. Next, the novel strategies for the design of device structures of blue PeLEDs are reviewed from the perspective of transport layers and interfacial layers. Accordingly, future directions for blue PeLEDs are discussed. This review can be a guideline for optimizing perovskite film and device structure of blue PeLEDs, thereby enhancing their development and application scope.

Keywords: perovskite light emitting diodes; perovskite film; device structure; blue LEDs

Li ZT, Cao K, Li JS, Tang Y, Ding XR et al. Review of blue perovskite light emitting diodes with optimization strategies for perovskite film and device structure. Opto-Electron Adv 4, 200019 (2021).

\section{Introduction}

Recently, metal halide perovskites have drawn considerable attention for their distinguished advantages, including high charge carrier mobility ${ }^{1}$, long carrier diffusion length ${ }^{2}$, large absorption coefficient ${ }^{3}$, low trap density ${ }^{4}$, and solution processability. All these advantages have prompted perovskites to be extensively researched in the fields of solar cells ${ }^{5}$, photodetectors ${ }^{6}$, and lasers ${ }^{7}$. Inspiring breakthroughs have been reported over several years for perovskite-based devices. For instance, perovskite solar cells have achieved a rectified power conversion efficiency of approximately $25.2 \%$, which makes it the fastest growing solar cell technology ${ }^{8}$. As direct bandgap semiconductors, perovskites are also an ideal light emitting material with excellent optoelectronic properties, in- cluding tunable bandgap, high color purity, wide color gamut, and high photoluminescence quantum yield (PLQY). Moreover, their bi-polar injection property allows them to be not only down-conversion materials ${ }^{9,10}$, but also be used in electroluminescent (EL) light emitting diodes ${ }^{1-13}$. Therefore, it is convincing that perovskite light emitting diodes (PeLEDs) have great potential in the fields of lighting and high-end display.

Since Tan et al. reported the first room-temperature EL PeLEDs ${ }^{14}$, the luminance and external quantum efficiency (EQE) of red and green PeLEDs have been tremendously improved, thus increasing their applicability to be on parallel with commercial OLEDs. To improve the luminance and efficiency of green and red PeLEDs, one aspect is to improve the quality of perovskite films.

${ }^{1}$ National \& Local Joint Engineering Research Center of Semiconductor Display and Optical Communication Devices, South China University of Technology, Guangzhou 510640, China; ${ }^{2}$ Foshan Nationstar Optoelectronics Company Ltd., Foshan 528000, China.

*Correspondence: ZC Li, E-mail: meztli@scut.edu.cn; JS Li, E-mail: jiasli@foxmail.com

Received: 20 June 2020; Accepted: 4 August 2020; Published: 28 February 2021 
High-quality perovskite films are composed of perovskites, which simultaneously have high exciton binding energy $\left(\mathrm{E}_{\mathrm{B}}\right)$, high conductivity, and low defect densities. For example, to improve the quality of a perovskite film, Li et al. doped it with insulation material polymer polyimide (PI) to fill the pinholes among the perovskite grains and effectively passivate the grain boundaries ${ }^{15}$. $\mathrm{Yu}$ et al. blended the ionic-conductive polymer of poly(ethylene oxide) (PEO) into perovskite precursors and successfully improved the film morphology without sacrificing the conductivity of the perovskite film ${ }^{16}$. Sargent et al. doped the bulk organic amino cation phenylethylammonium $\left(\mathrm{PEA}^{+}\right)$into perovskite films and fabricated perovskite films with multi quantum well (MQW) structure for the first time. Near-infrared PeLEDs based on the MQW structure have shown a high EQE of $8.8 \%{ }^{17}$. Moreover, the morphology texture of perovskite films has been proven as a key factor influencing device performance. While dense and uniform perovskite films are beneficial for improving the contact between the carrier transport layers and diminishing the leakage current, some novel morphology textures, such as sub-micro concave structures, can improve the light outcoupling performance of PeLEDs ${ }^{18}$.

Another way to optimize the EL performance of green and red PeLEDs is to finely design the device structure. For example, Wang et al. selected the polyethyleneimine (PEI)-modified low work function metal oxide $\mathrm{ZnO}$ and high hole mobility polymer (poly [(9,9-dioctylfluorenyl2,7-diyl)-co-(4,40-(N-(4-sec-butylphenyl diphenylamine)]) (TFB) as the electron transport layer (ETL) and hole transport layer (HTL) materials, respectively and fabricated high luminance red PeLEDs with a novel inversed device structure ${ }^{19}$. Shi et al. designed a novel insulator-perovskite-insulator device structure for the first time without using the commonly used hole injection material PEDOT:PSS, because PEDOT:PSS has proven to be negatively affecting device stability due to its acidity and extensive nonradiative channels ${ }^{20}$. The first high-efficiency PeLEDs were reported by Cho et al., who applied a synergetic modification strategy for both perovskite film and device structure, and the optimized green PeLEDs showed a maximum EQE of $8.53 \%{ }^{21}$. Currently, the EQEs of red and green PeLEDs exceed $20 \%{ }^{22-24}$.

In addition to red and green PeLEDs, the fabrication of blue PeLEDs is also a key factor in the fields of lighting and display. To obtain white PeLEDs, light with two or more different colors must be mixed ${ }^{25,26}$. In the fields of lighting and backlight display, white light can be obtained by using blue LEDs to stimulate down-conversion materials ${ }^{27-29}$. In the field of full-color display, as blue is one of the three-primary colors of light, the fabrication of EL blue LEDs is a prerequisite for fabricating RGB LED devices ${ }^{30,31}$. However, when compared with red and green PeLEDs that have had continuous progress, the development of the luminance and efficiency of blue PeLEDs is lagging far behind. The reason can be explained from two aspects. In terms of blue-emitting perovskite films, the larger bandgap of blue perovskites promotes the formation of sub-bandgap trap energy levels, which can not only lead to nonradiative recombination but also have a negative effect on the color purity $^{32}$. In terms of device structure, the deeper valanced band maximum (VBM) and higher conduction band minimum (CBM) energy levels of blue perovskites compared with those of red and green ones lead to a larger energy offset at the interface of the emitting material layer (EML) and charge transport layers (CTLs), which can severely affect the charge injection efficiency ${ }^{33}$. Therefore, it is necessary to achieve both high-quality perovskite films and proper design of device structures for blue PeLEDs.

In this review, we categorize the recent progress of blue PeLEDs in terms of composition of perovskite films and summarize the main challenges and corresponding optimization strategies. Next, we review the novel strategies for the design of the device structure of blue PeLEDs from the perspective of transport layers and the interface of the EML and CTLs. Finally, future directions for blue PeLEDs are analyzed.

\section{Challenges and optimization strategies for perovskite films of blue PeLEDs}

\section{Pure 3D perovskites}

Three-dimensional (3D) metal halide perovskites are defined by a chemical formula of $\mathrm{ABX}_{3}$, where the A site refers to the monovalent cations, including the organic cations $\left(\mathrm{MA}^{+}, \mathrm{FA}^{+}\right)$and inorganic cation $\left(\mathrm{Cs}^{+}, \mathrm{Rb}^{+}\right)$, the $\mathrm{B}$ site refers to the divalent cations $\left(\mathrm{Pb}^{2+}, \mathrm{Sn}^{2+}, \mathrm{Ti}^{2+}\right)$, and the $\mathrm{C}$ site refers to the halide ions $\left(\mathrm{I}^{-}, \mathrm{Br}^{-}, \mathrm{Cl}^{-}\right)$. A divalent cation, such as $\mathrm{Pb}^{2+}$, can form an ionic bond with the halide ions and form a periodically aligned octahedron structure $\left[\mathrm{PbX}_{6}\right]$; while a monovalent cation can insert into the intervals between $\left[\mathrm{PbX}_{6}\right]^{-}$to reach charge 
conversation and stabilize the crystal structure (Fig. 1(a)). As for $3 \mathrm{D}$ perovskites, the CBM energy level is dependent on the p-orbital of the divalent cation, while the VBM energy level is related to the p-orbital of the halide ions. By increasing the ratio of $\mathrm{Cl}^{-}$, the $\mathrm{VBM}$ is downshifted and the bandgap of perovskite can be enlarged ${ }^{34}$. In 2015, Kumawat et al. used $\mathrm{MABr}$ and $\mathrm{PbCl}_{2}$ as precursors and obtained the first blue PeLEDs. The bandgap of $3 \mathrm{D}$ perovskites can be turned from $2.42 \mathrm{eV}$ to $3.16 \mathrm{eV}$ by increasing the ratio of $\mathrm{Cl}^{-}$(Fig. 1(b)). However, the EQE of blue PeLEDs is extremely low $(0.0001 \%)$ due to the poor film morphology and large grain size $(10-20 \mu \mathrm{m})$ (Fig. 1(c) $)^{35}$. Sadhanala et al. replaced the low solubility precursor $\mathrm{PbCl}_{2}$ with $\mathrm{Pb}(\mathrm{COOH})_{2}$ and increased the concentration of $\mathrm{Cl}^{-}$by adding $\mathrm{MACl}$. Blue PeLEDs with $\mathrm{MAPbCl}_{3}$ as the emissive layer exhibited an EL at $77 \mathrm{~K}$ with a peak wavelength at $427 \mathrm{~nm}$ (Fig. 1(d)). The extremely low PLQY $(<1 \%)$ caused by the large number of defect sites eliminated the EL of PeLEDs operated at room temperature ${ }^{36}$. Therefore, the poor film morphology as well as defect sites are the main challenges for the further development of blue PeLEDs with pure 3D perovskite films as the emissive layer. In this section, the strategies for morphology modification and defect passivation of pure $3 \mathrm{D}$ perovskite films are reviewed.

\section{Morphology optimization strategies}

In general, dense and uniform perovskite films with few pinholes are beneficial for the elimination of leakage current, which is essential for the enhancement of EQE and reduction of Joule heat. The morphology can be modified by adjusting the molar ratio of perovskite precursors. Wang et al. fabricated dense and uniform polycrystalline $\mathrm{MAPb}\left(\mathrm{Br}_{1-x} \mathrm{Cl}_{x}\right)_{3}$ films by increasing the molar ratio of $\mathrm{MA}^{+}$and decreasing the whole concentration of the precursors. The results suggested that the small perovskite grain size as well as the thin perovskite film thickness are crucial for facilitating the radiative recombination rate. Optimized sky-blue (490 nm) PeLEDs showed a maximum luminance of $154 \mathrm{~cd} / \mathrm{m}^{2}$ at $4.1 \mathrm{~V}^{37}$. Mixing the A site cations is also an effective way to improve the film morphology. Kim et al. fabricated
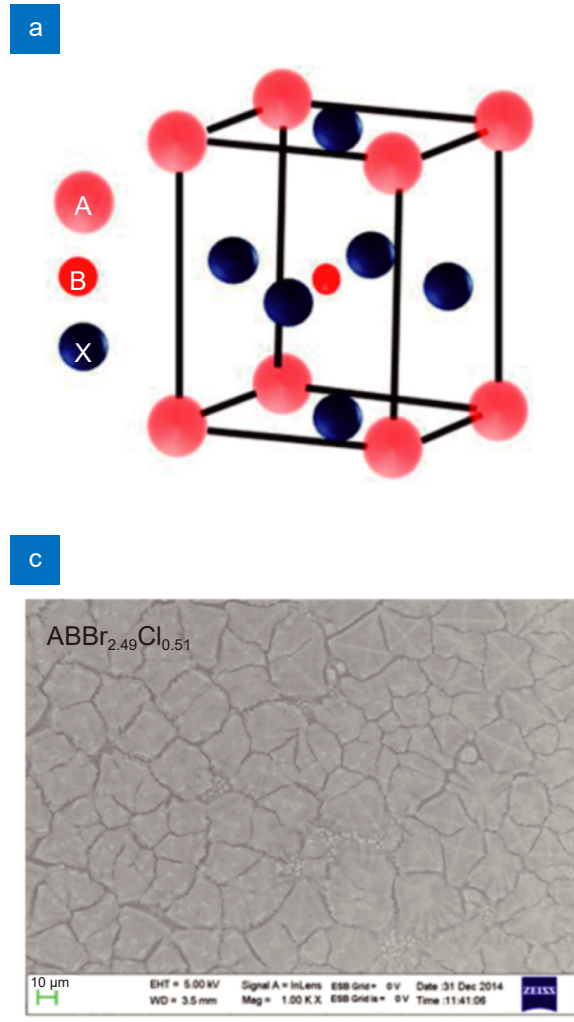

b

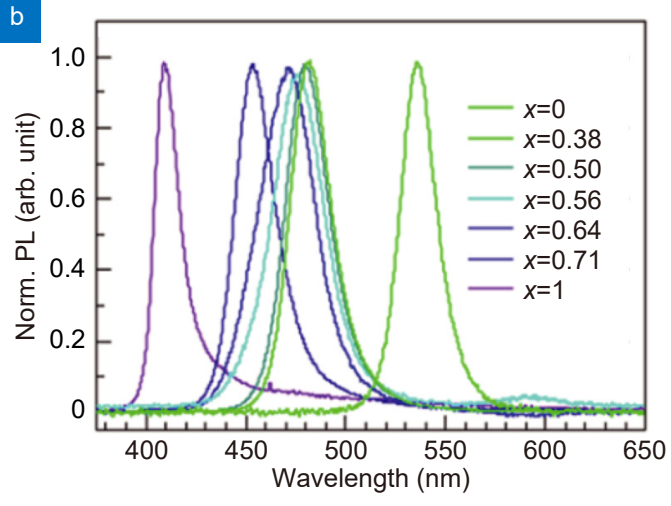

d

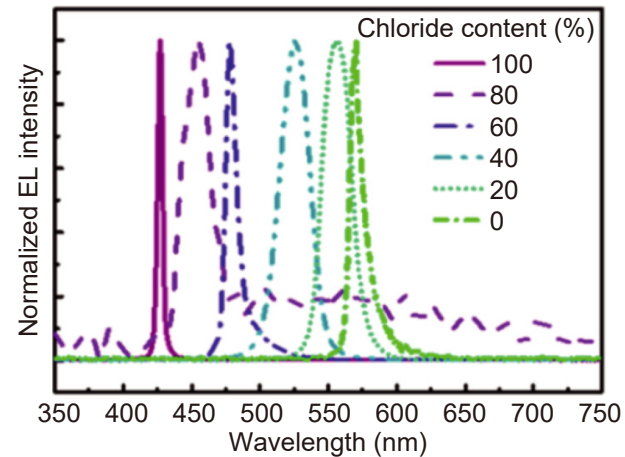

Fig. 1 | Preliminary study of pure 3D perovskites and their blue PeLEDs. (a) Schematic diagram of the structure of 3D perovskites. (b) Photoluminescence spectra of $\mathrm{MAPb}\left(\mathrm{Br}_{1-x} \mathrm{Cl}_{x}\right)_{3}$ perovskite film with different ratios of $\mathrm{Cl}$. (c) SEM image of $\mathrm{MAPb}\left(\mathrm{Br}_{1-x} \mathrm{Cl}_{x}\right)_{3}$ perovskite film on ITO/PEDOT:PSS substrate. (d) Normalized EL spectra of $\mathrm{CH}_{3} \mathrm{NH}_{3} \mathrm{~Pb}\left(\mathrm{Br}_{x} \mathrm{Cl}_{1-x}\right)_{3}[0 \leq x \leq 1]$ perovskite thin-film-based LEDs with different chloride-bromide ratios, as indicated and measured at $77 \mathrm{~K}$. Figure reproduced with permission from: (a-c) ref. ${ }^{35}$, American Chemical Society; (d) ref. ${ }^{36}$, American Chemical Society. 
tri-cation $\left(\mathrm{MA}^{+}, \mathrm{FA}^{+}\right.$, and $\left.\mathrm{Cs}^{+}\right)$3D perovskite films and modified the film morphology by adjusting the molar ratio between those cations. Similarly, the results suggested that the film thickness as well as the grain size showed considerable influence on the EL performance of blue PeLEDs. A suitable amount of $\mathrm{Cs}^{+}$is important because it can not only improve the stability of perovskite films but also modify the film morphology. However, when adding an excess amount of $\mathrm{Cs}^{+}$, rod-like shaped perovskite grains were formed and mainly existed on the surface of perovskite film, which hindered the carrier injection. The fabricated blue PeLED (475 nm) exhibited a maximum luminance of $3567 \mathrm{~cd} / \mathrm{m}^{2}$ with an EQE of $1.7 \%{ }^{38}$.

All inorganic metal halide perovskites show superior thermal stability compared with their organic counterparts. Wang et al. doped $\mathrm{RbBr}$ and $\mathrm{RbCl}$ into the $\mathrm{CsPb}$ $\mathrm{Br}_{3}$ precursor solution. By adjusting the ratios of $\mathrm{Rb}^{+}, \mathrm{Br}$ and $\mathrm{Cl}^{-}$, they built up sky-blue $(492 \mathrm{~nm})$, blue $(473 \mathrm{~nm})$ and deep-blue $(468 \mathrm{~nm})$ PeLEDs. The radius of $\mathrm{Rb}^{+}$ $(161 \mathrm{pm})$ is smaller than that of $\mathrm{Cs}^{+}(174 \mathrm{pm})$ and the incorporation of $\mathrm{Rb}^{+}$into the $\mathrm{CsPbBr}_{3}$ enlarges the bandgap of perovskites and causes the blue shift of the emission spectra. The film morphology can also be improved by $\mathrm{Rb}^{+}$incorporation to modulate the nucleation process. The lower solubility of RbX compared with CsX facilitated the initial precipitation of RbX small crystals, which can act as nucleation cores that increase the number of perovskites and decrease the size of as-formed perovskite grains. The coverage of perovskite films increased from $80 \%$ to $99 \%$ (Fig. 2(a)). The maximum luminances of sky-blue $(492 \mathrm{~nm})$ and blue $(473 \mathrm{~nm})$ PeLEDs are $244 \mathrm{~cd} / \mathrm{m}^{2}$ and $112 \mathrm{~cd} / \mathrm{m}^{2}$, respectively. It is worth mentioning that all $\mathrm{RbX}$-incorporated blue PeLEDs exhibit great EL spectral stability, indicating improved structural stability by $\mathrm{RbX}$ incorporation. However, the low PLQY $(<0.25 \%)$ of Rb-Cs alloy perovskite films limits the further improvement of device efficiency ( $\mathrm{EQE}=0.062 \%$ for blue device), which originates from the increased number of grain boundaries that facilitate the nonradiative recombination rate ${ }^{39}$.

\section{Defect passivation strategies}

Although 3D perovskites have exhibited outstanding conductivity, the low PLQY of pure 3D perovskite films caused by the large number of defect sites inside the grains or at the grains boundaries limits the further improvement of device performance. As mentioned above, $\mathrm{Rb}^{+}$-incorporated pure 3D perovskite films show good film morphology but low PLQY due to the high defect density at grain boundaries. To diminish the defect density and improve the PLQY of $\mathrm{Rb}^{+}$-incorporated perovskite films, Yuan et al. proposed a cocktail cation strategy that incorporates four kinds of monovalent cation, $\mathrm{Rb}^{+}, \mathrm{Fa}^{+}, \mathrm{PEA}^{+}$, and $\mathrm{K}^{+}$, into $\mathrm{CsPbBr}{ }_{x} \mathrm{Cl}_{3-x}$ perovskites and fabricated sky-blue $(484 \mathrm{~nm})$ PeLEDs with an improved EQE of $2.01 \%$. Also, the EL spectra remain stable under different bias voltage, indicating a suppressed halogen migration (Fig. 2(b)). The results showed that $\mathrm{Rb}^{+}$can play an important role in optimizing the film morphology with increased coverage and decreased surface roughness, while $\mathrm{K}^{+}$and $\mathrm{PEA}^{+}$can form a passivation layer outside the grains to suppress the nonradiative recombination, leading to an improved PLQY from $0.94 \%$ to $17.1 \%{ }^{40}$. In addition to the A site mixing strategy, the B site mixing strategy can also hinder the formation of defect sites and improve the PLQY of perovskite films. Gangishetty et al. incorporated $\mathrm{Mn}^{2+}$ into stoichiometrically prepared $\mathrm{Cs}_{0.4} \mathrm{MA}_{0.6} \mathrm{PbBr}_{1.9} \mathrm{Cl}_{1.1}$ precursor solution and partly substituted the $\mathrm{Pb}^{2+}$ with $\mathrm{Mn}^{2+}$ in as-formed 3D perovskite films. Sky-blue PeLEDs were fabricated with a maximum luminance of $1470 \mathrm{~cd} / \mathrm{m}^{2}$ and EQE of $0.58 \%$. The results suggested that $\mathrm{Mn}^{2+}$ incorporation can not only improve the crystallinity and film morphology but also diminished the formation of defect sites inside the perovskite grains. $\mathrm{Mn}^{2+}$-incorporated pure 3D perovskite films exhibited an improved film PLQY from $\sim 0 \%$ to $6 \%$ (Fig. 2(c)). Moreover, the $\mathrm{Mn}^{2+}$ incorporated PeLED showed greatly improved EL spectral stability as well as prolonged device operating lifetime $\left(L_{50}=24 \mathrm{~min}\right.$ at $\left.3 \mathrm{~mA} / \mathrm{cm}^{2}\right)$, indicating that $\mathrm{Mn}^{2+}$ can reduce the mobility of $\mathrm{Br}^{-}$and $\mathrm{Cl}^{-}{ }^{41}$. Du et al. fabricated deep-blue 3D perovskite films with high PLQY by a thermal evaporation method. $\mathrm{CsCl}$ and $\mathrm{PbBr}_{2}$ were selected as the source materials for thermal evaporation. When the substrates remained static, the obtained $\mathrm{CsPbBr}{ }_{x} \mathrm{Cl}_{3-x}$ films could be divided into several regions by the peak wavelength of PL spectra from deep-blue ( $450 \mathrm{~nm}$, close to $\mathrm{CsCl}$ source) to sky-blue ( $480 \mathrm{~nm}$, close to $\mathrm{PbBr}_{2}$ source). The deep-blue 3D perovskites films showed an optimal PLQY of $18.7 \%$, which is the highest PLQY value ever reported for a 3D perovskite film (Fig. 2(d)). Such enhancement of PLQY originated from the formation of $\mathrm{Cs}_{4} \mathrm{PbBr}_{6}$ phase due to excess amounts of $\mathrm{CsCl}$. The $\mathrm{Cs}_{4} \mathrm{PbBr}_{6}$ phase can passivate the defect site at the grain boundaries of $\mathrm{CsPbBr}{ }_{x} \mathrm{Cl}_{3-x}$. However, the excess amounts of $\mathrm{Cs}_{4} \mathrm{PbBr}_{6}$ lower the conductivity of 

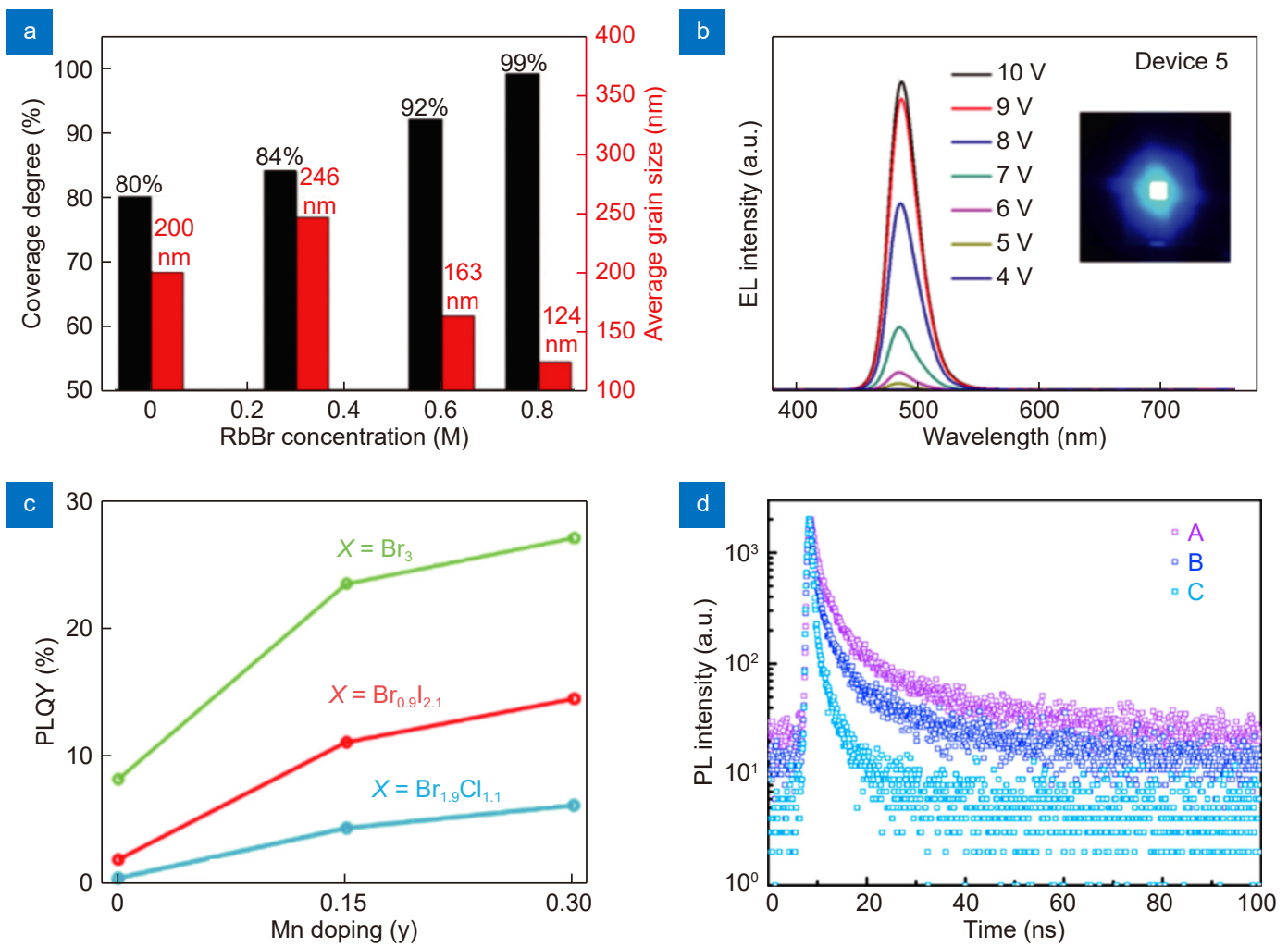

Fig. 2 | Morphology and PLQY modification for 3D perovskites and their blue PeLEDs. (a) The calculated coverage degree and average grain size of perovskite films with various $\mathrm{CsBr}: \mathrm{RbBr}$ molar ratios. (b) EL spectra of the device fabricated with cocktail cation strategy operating under different voltages. The inset shows a digital photograph of a device in operation. (c) PLQY measurements with various Mn doping ratios. (d) Time-resolved photoluminescence (TRPL) decays of samples A (deep-blue region), B (blue region), and C (sky-blue region). Figure reproduced with permission from: (a) ref..$^{39}$, Royal Society of Chemistry; (b) ref. ${ }^{40}$, American Chemical Society; (c) ref. ${ }^{41}$, American Chemical Society; (d) ref. ${ }^{42}$, American Chemical Society.

perovskite films, which hinders the current injection rate. The maximum EL intensities of deep-blue, blue, and sky-blue devices are $51 \mathrm{~cd} / \mathrm{m}^{2}, 121 \mathrm{~cd} / \mathrm{m}^{2}$, and $87 \mathrm{~cd} / \mathrm{m}^{2}$, respectively, which originates from the synergistic effect of $\mathrm{Cs}_{4} \mathrm{PbBr}_{6}$ passivation and the degraded current injection $^{42}$.

Therefore, even though 3D perovskite films exhibited some excellent properties such as solution processability and high conductivity, the large number of defect sites as well as the low exciton bonding energy limits further improvement of the PLQY. Besides, the low solubility of $\mathrm{PbCl}_{2}$ limits the degree of hypsochromic shift of the emission spectra. Based on the current research findings, several strategies should be considered for the further optimization of pure 3D perovskites films: 1) Adopting the A site mixing strategy. It has been demonstrated that mixing A site cations can promote the device performance through improving the film morphology and slowing down the halogen mobility. Based on that, the category and the molar ratio of the selected A site cations should be thoroughly considered. 2) Adoping $\mathrm{O}$ atoms contained passivation agents. Except for the commonly used amino-functionalized moieties, $\mathrm{O}$ atoms contained passivation agents should also be exploited as the inductive effect caused by $\mathrm{O}$ atoms has been proved to be beneficial for improving the passivation efficiency. 3) Adopting $\mathrm{Cl}$-contained doping agents. In order to fabricate stable blue and deep-blue 3D PeLEDs, the $\mathrm{Cl}$ concentration in the final 3D perovskite films should be raised. $\mathrm{Cl}-$ contained agents such as the organic chlorines can be suitable for increasing the molar ratio of $\mathrm{Cl}$ through either doping into the precursor solution or post treatment.

\section{Colloidal perovskite quantum dots (PQDs)}

Compared with pure 3D perovskites, colloidal perovskite quantum dots (PQDs) possess advantages including high $E_{\mathrm{B}}(\sim 40 \mathrm{meV})$, low defect density, and high environmental stability. All inorganic $\mathrm{CsPbX}_{3}$ QDs show better water and thermal stability compared with their organic counterparts, which are the most widely researched PQDs. As the PLQYs of PQDs dispersed in 
solution have reached up to $\sim 100 \%$, PeLEDs with PQDs as the emissive layer may have higher EQE theoretically. Similar with pure 3D blue perovskites, most blue PQDs are synthesized by a mixed halides strategy due to the low solubility of $\mathrm{PbCl}_{2}$ in polar solvent. In 2015, Song et al. synthesized all inorganic blue $\mathrm{CsPbBr}_{x} \mathrm{Cl}_{3-x}$ QDs by the hot-injection method. The as-synthesized PQDs emitted at $455 \mathrm{~nm}$ with PLQY up to $60 \%$ in dispersion. Moreover, PQD-based blue PeLEDs were fabricated for the first time with a maximum luminance of $742 \mathrm{~cd} / \mathrm{m}^{2}$ and EQE of $0.07 \%{ }^{43}$. Such low EQE can be explained from four aspects. First, the excess number of insulated long-chain alkyl ligands in PQD dispersion decreases the conductivity of PQD films. Although the concentration of ligands in dispersion could be diminished by increasing the length of the purification process, it could not be fully diminished as the purification process can cause damage to the PQDs. Secondly, PQD films usually exhibit poor film morphology with huge leakage current. Thirdly, the low solubility of $\mathrm{PbCl}_{2}$ limits the tuning range of the emission spectra. Although the molar ratio of $\mathrm{Cl}^{-}$can be increased by some other strategy, such as ion exchange, the stability as well as the PLQY of PQDs decreases as the molar ratio of $\mathrm{Cl}^{-}$increases, which is due to the weak interaction between the $\mathrm{Cl}^{-}$and the ligand. Finally, the PLQY of PQDs in thin films is far behind that in dispersion because of the ligand loss phenomenon, which facilitates the formation of surface defects that cause nonradiative recombination. In short, there are four main challenges that limit the further improvement of PQD-based PeLEDs: low conductivity of PQD films, poor film morphology, narrow spectrum tuning range, and large number of defect sites. In this section, several strategies are reviewed to solve the above four challenges.

\section{Conductivity optimization strategies}

To improve the conductivity of PQD films, excess amounts of insulated ligands should be diminished without lowering the PLQY of PQDs. Pan et al. adopted a ligand exchange strategy to improve the conductivity of PQD films. The halide ion-pair ligand of di-dodecyl dimethyl ammonium bromide (DDAB) was added to the dispersion to thoroughly substitute the long-chain oleic acid ligands at the surface of PQDs. Such a ligand exchange process can be triggered because of the stronger affinity between $\mathrm{Br}^{-}$and the positive site $\left(\mathrm{Cs}^{+}\right.$and $\left.\mathrm{Pb}^{2+}\right)$ compared with the oleic group as well as the stronger affinity of $\mathrm{DDA}^{+}$with the negative sites $(\mathrm{Br}-)$ on the $\mathrm{PQD}$ surface. The shorter chain length of $\mathrm{DDA}^{+}$successfully improved the conductivity of PQD films. By substituting the DDAB with the mixed halide-ion-pair ligand di-dodecyl dimethyl ammonium bromide chloride (DDAB$\mathrm{rCl}$ ), sky-blue (490 nm) PQD-based PeLEDs were fabricated with maximum EQE of $1.9 \%{ }^{44}$. The conductivity of PQD films can be further improved by introducing an alkali metal halide as ligands to partly substitute the organic ligands. Yang et al. introduced $\mathrm{K}_{2} \mathrm{CO}_{3}$ into the precursor solution, and $\mathrm{K}^{+}$was found to partly occupy the surface vacancies of PQDs; stable blue $\mathrm{CsPb}\left(\mathrm{Br}_{x} / \mathrm{Cl}_{1-x}\right)_{3}$ QDs with a peak wavelength of $477 \mathrm{~nm}$ were synthesized. The desorption of the organic ligands (DDAB and OTAc) at the surface of PQDs was observed, and the $\mathrm{K}^{+}$ acted as inorganic ligands capping at the surface of PQDs by forming ionic bonding with the halide ions. When the ratio of $\mathrm{K} / \mathrm{Pb}$ was $4 \%$, blue PeLEDs based on the $\mathrm{K}^{+}$modified PQDs showed a maximum luminance of $212.9 \mathrm{~cd} / \mathrm{m}^{2}$ and EQE of $1.96 \%{ }^{45}$. Yao et al. provided a novel short-chain complex agent to speed up the ligand exchange process. The calcium-tributylphosphine oxide (Ca-TBPO) complexes was added into the $\mathrm{CsPb}\left(\mathrm{Br}_{x} / \mathrm{Cl}_{1-x}\right)_{3}$ QDs dispersion, and the long-chain organic ligands was confirm to be replaced by the short-chain TBPO and the $\mathrm{Ca}^{2+}$ cations due to their good solubility in non-polar organic solvents. The single-carrier device measurement confirmed a synergistically improved electron current and hole current injection. Based on this strategy, deep blue (463 nm) PeLEDs with maximum EQE up to 3.3\% was achieved ${ }^{46}$. More recently, Dong et al. provided a novel bipolar-shell resurfacing method to synthesize PQDs with higher conductivity. The surface of the bipolar-shell $\mathrm{PQDs}$ are composed of a negatively charged inner Br-riched shell and a positively charged outer shell composed of $\mathrm{Na}^{+}$cations and polar solvent molecules. The insulated oleate ligands in the pristine PQDs was confirmed to be largely substituted, with the diminished inner distance $(<2 \AA)\left(1 \AA=10^{-10} \mathrm{~m}\right)$ of the PQDs in the solid films that enable more efficient charge transport among PQDs. With the improved charge mobility $\left(\geq 0.01 \mathrm{~cm}^{2} \mathrm{~V}^{-1} \mathrm{~s}^{-1}\right)$ of the PQD films, the EQE of the blue (479 $\mathrm{nm}$ ) PeLED based on the bipolar-shell PQDs reached a maximum of $12.3 \%$, which is one of the most efficient blue PeLED ${ }^{47}$.

\section{Morphology optimization strategies}

The poor morphology of PQD films can be explained from two aspects. On the one hand, the long-chain 
ligands, such as oleic acid (OA) and oleylamine (OAm), show poor affinity to the PQD surface, which leads to severe ligand loss during the purification and film formation process. Such a ligand loss phenomenon leads to poor coverage of PQD films due to the weakened dispersibility of PQDs, which can cause severe agglomeration. This ligand loss phenomenon can be even worse in blue-emitting PQDs due to the poor affinity of ligands with $\mathrm{Cl}$. On the other hand, the concentration of PQDs in dispersion is too low to form a dense and uniform perovskite film, so the leakage current of $\mathrm{PQD}$-based PeLEDs can be further increased. To optimize the morphology of PQD films, Li et al. applied a vapor-based crosslinking strategy that treated PQD films with trimethylaluminum (TMA) vapor ${ }^{48}$. The resulting film exhibited nearly complete surface coverage thanks to the crosslinking effect of TMA. However, the strongly reactive TMA vapor can cause damage to the chloride-containing perovskites. The as-prepared sky-blue $(480 \mathrm{~nm})$ PeLEDs exhibited a maximum luminance of only 8.7 $\mathrm{cd} / \mathrm{m}^{2}$. As the agglomeration of PQDs is harmful to the morphology of PQD films, the affinity between ligands and the PQD surface should be strengthened. Ochsenbein et al. synthesized deep-blue (463 nm) PQDs capped with zwitterionic sulfobetaine ligands. The PQDs with sulfobetaine ligands showed improved dispersity and allowed for a more thorough purification of the PQD dispersion due to the improved affinity. The PLQY of PQDs in dispersion remained nearly unchanged after three times purification and remained at $\sim 90 \%$ after four times purification by ethyl acetate. The film morphology, EQE, and reproducibility of PeLEDs based on the zwitterionic sulfobetaine ligand-capped PQDs can be further improved by additional doped polystyrene $(1 \mathrm{mg} / \mathrm{ml})^{49}$.

In addition to the modification of ligand affinity, some novel film formation technologies can also be helpful for the improvement of blue PQD film morphology. Deng et al. noted that the main reason for the high surface roughness and excessive pinholes of PQD films originates from the relatively low concentration of PQDs in dispersion $(\sim 0.5 \mathrm{mg} / \mathrm{ml})$. By applying a dip-coating method, dense and uniform blue PQD films were formed due to the optimized film formation process. High luminance (2473 \pm $105 \mathrm{~cd} / \mathrm{m}^{2}$ ) deep-blue (445 nm) PeLEDs were fabricated with an average EQE of $1.18 \%$ thanks to the improved film morphology $y^{50}$. In addition to ligand affinity, the polarity and boiling point significantly influence the film morphology. Yao et al. dispersed $\mathrm{CsPbBr}_{x} \mathrm{Cl}_{3-x}$ QDs by toluene/hexane mixed solvents and successfully fabricated pinhole-free PQD films due to the improved dispersity as well as weakened agglomeration of PQDs in films. The lower polarity of hexane (0.1) compared to toluene (2.4) is beneficial for PQD dispersity, while the higher boiling point of toluene $\left(110{ }^{\circ} \mathrm{C}\right)$ compared with hexane $\left(68{ }^{\circ} \mathrm{C}\right)$ reduces the variation of concentration of PQDs during the film formation process and improves the size homogeneity of PQDs ${ }^{51}$. Li et al. proposed a novel heterogeneous nucleation method that can effectively increase the concentration of PQDs in dispersion. Microscale $\mathrm{SiO}_{2}$ spheres $(2.32 \mu \mathrm{m}$ in diameter) were added to the precursor solution to reduce the crystalline formation energy and increase the concentration of PQDs in dispersion. The low surface energy as well as the inert property of microscale $\mathrm{SiO}_{2}$ spheres can facilitate the heterogeneous distribution of PQDs on the surface of $\mathrm{SiO}_{2}$ spheres and reduce the agglomeration of PQDs in dispersion $^{52}$.

\section{Spectra tuning strategies}

The traditional strategies for spectra tuning include adjusting the ratio of halides in precursor solution and the halogen exchange. Shin et al. applied a ligand-mediated post-treatment strategy by using both DDAB and DDAC as the ligands to synthesize blue PQDs. Different ratios of the DDAB and DDAC ligands were added to the dispersion to control the degree of halogen exchange by adjusting the concentration of $\mathrm{Cl}^{-}$. Blue PeLED based on this DDAB/DDAC modified PQDs was fabricated (Fig. 3(a)), and the EL spectra could be adjusted from $460 \mathrm{~nm}$ to $480 \mathrm{~nm}^{53}$. Furthermore, Shynkarenko et al. applied an in-situ ligand-mediated strategy to synthesize PQDs with DDAB and DDAC as ligands in the precursor. By varying the ratios of $\mathrm{DDAB}$ and DDAC in the precursor solution, the emission spectra could be adjusted in a wide range of $460-510 \mathrm{~nm}$ (Fig. 3 (b)). As the $\mathrm{Cl}^{-}$ concentration is usually limited by the poor solubility of $\mathrm{PbCl}_{2}$ in precursor solution, DDAC was used as an efficient $\mathrm{Cl}$ source due to the higher solubility of DDAC in precursor solution. However, the operating lifetime $\left(T_{50}\right)$ of the blue PeLEDs is only $26 \mathrm{~s}$ with an initial luminance of $100 \mathrm{~cd} / \mathrm{m}^{2}{ }^{54}$. Ye et al. provided a novel halogen exchange enhancement strategy by using different categories of benzenesulfonates to determine the direction and degree of halogen exchange in PQD dispersion (Fig. $3(\mathrm{c}))$. By adjusting the concentration of tetrabutylammonium (TBSA), the dislocated $\mathrm{Cl}^{-}$in dispersion 


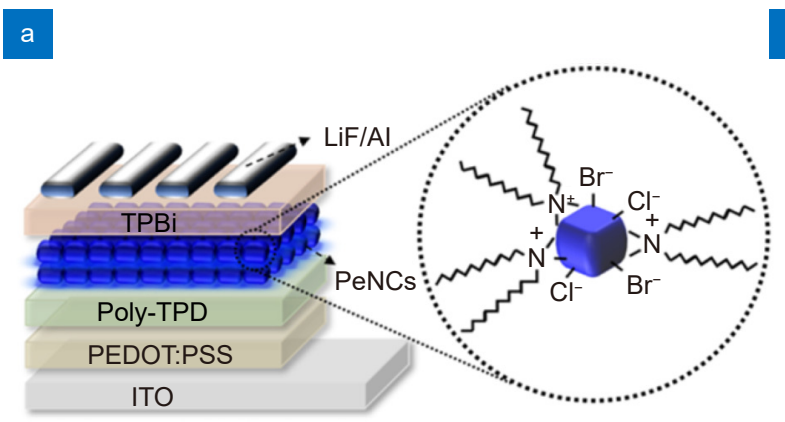

b
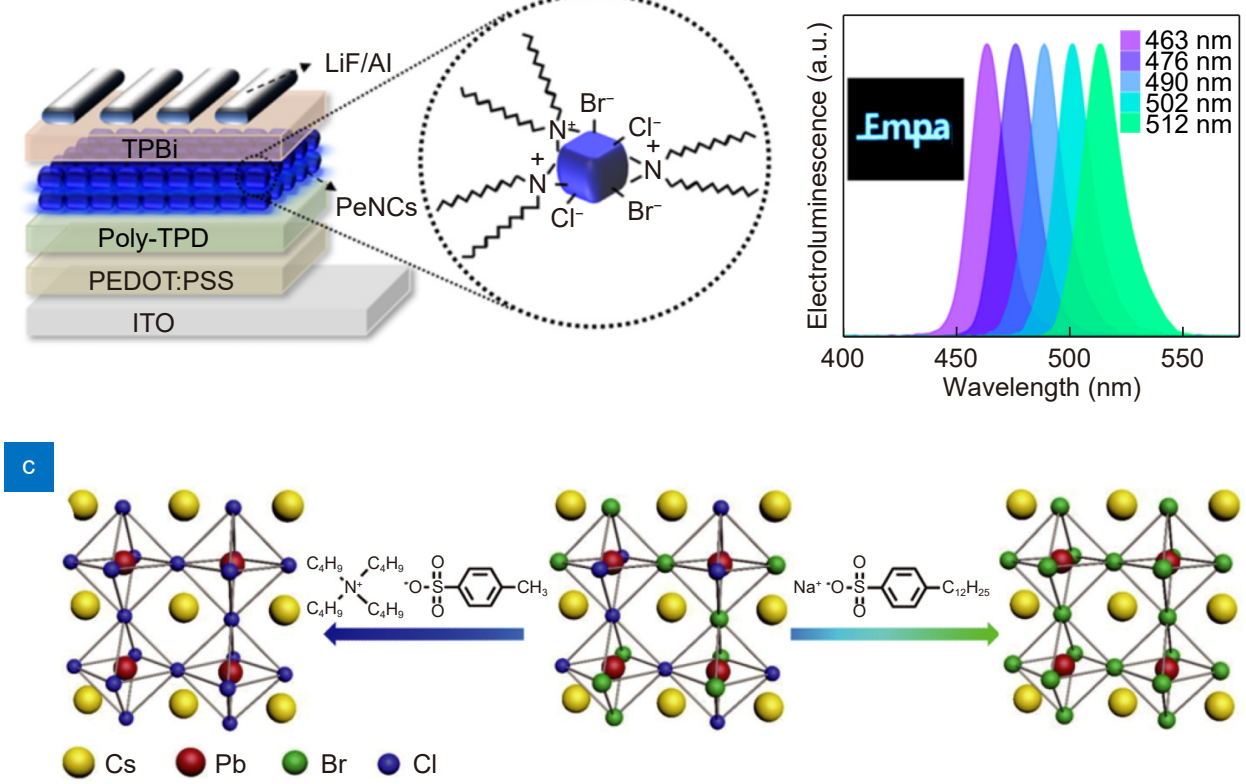

Fig. 3 | Spectral tuning strategies for PQDs and their blue PeLEDs. (a) Schematic diagram of $\mathrm{CsPbBr}_{x} \mathrm{Cl}_{3-x}$ QD-based blue PeLED device structure by the DDAB/DDAC post-treatment strategy. (b) EL spectra of $\mathrm{CsPbBr}_{x} \mathrm{Cl}_{3-x}$ QD-based PeLEDs with various ratios of DDAB and DDAC in precursor solution. The inset shows a digital photograph of the device in operation. (c) Schematic diagram of the halogen exchange process in PQDs enhanced by benzenesulfonates. Figure reproduced with permission from: (a) ref. ${ }^{53}$, American Chemical Society; (b) ref. ${ }^{54}$, American Chemical Society; (c) ref. ${ }^{55}$, American Chemical Society.

facilitated substitution with $\mathrm{Br}^{-}$in $\mathrm{CsPbBr}_{1.5} \mathrm{Cl}_{1.5}$ QDs and finally achieved completed halogen exchange with the PL peak wavelength blue-shifted from $475 \mathrm{~nm}$ to 409 nm. Based on this facile strategy, PQD-based blue (469 $\mathrm{nm})$ and sky-blue (496 nm) PeLEDs achieved maximum luminances of $30 \mathrm{~cd} / \mathrm{m}^{2}$ and $603 \mathrm{~cd} / \mathrm{m}^{2}$ with EQEs of $0.65 \%$ and $2.6 \%$, respectively ${ }^{55}$. It is worth noting that those benzenesulfonate-modified PeLEDs show stable EL spectra during operation, which is unusual in PeLEDs based on $\mathrm{Br} / \mathrm{Cl}$ mixing strategy. The $\mathrm{Rb}^{+}$incorporation is also a facile method to adjust the emission spectra. Todorovic et al. synthesized pure Br-based sky-blue (490 $\mathrm{nm}) \mathrm{Rb}_{x} \mathrm{Cs}_{1-x} \mathrm{PbBr}_{3}$ QDs with PLQY exceeding $90 \%$ in dispersion. Based on this Rb-based QD dispersion, skyblue PeLEDs with Rb-based PQDs as the emissive layer were fabricated for the first time, with maximum luminance and EQE of $183 \mathrm{~cd} / \mathrm{m}^{2}$ and $0.69 \%$, respectively $y^{56}$.

\section{Defect passivation strategies}

Previous research in the field of PQD synthesis has shown that the incorporation of metal ions, such as $\mathrm{K}^{+}$, $\mathrm{Rb}^{+}, \mathrm{Cd}^{+}, \mathrm{Ni}^{+}, \mathrm{Mn}^{2+}, \mathrm{Ca}^{2+}, \mathrm{Mg}^{2+}, \mathrm{La}^{3+}, \mathrm{Al}^{3+}$, and $\mathrm{Y}^{3+}$, into the precursor solution can diminish the generation of defect sites like halogen vacancies at the surface of PQDs by forming a passivation layer outside or hindering the gen- eration of structural defects inside the PQDs by partially doping into the crystal lattice ${ }^{45,56-62}$. Meng et al. proposed an FABr-modified multi-cation hot-injection method to diminish the defect density and promote PLQY. By adding proper amounts of $\mathrm{FABr}$ and $\mathrm{Rb}^{+}$into precursor solution, blue-greenish $(502 \mathrm{~nm})$ and deepblue $(466 \mathrm{~nm})$ PQDs were synthesized with PLQYs of $64.5 \%$ and $49.8 \%$ in solid film state, respectively. These results are because $\mathrm{FABr}$ and $\mathrm{Rb}^{+}$restrict the growth of PQDs and minimize the size of PQDs to only $4.79 \mathrm{~nm}$, which contributes to a stronger quantum confinement effect compared with the pristine ones. Thanks to the smaller size of PQDs, the $E_{\mathrm{B}}(221 \mathrm{eV}$ for $502 \mathrm{~nm}, 253 \mathrm{eV}$ for $466 \mathrm{~nm}$ ) is greatly increased, which is beneficial for the improvement of PLQY. Moreover, the FABr forms a passivation layer outside PQDs and significantly decreases the number of defect sites at the surface of PQDs, which is confirmed by the TRPL measurement (Fig. 4(a) $)^{63}$. Hou et al. doped $\mathrm{MnCl}_{2}$ into precursor solutions and synthesized $\mathrm{CsMn}_{y} \mathrm{~Pb}_{1-y}(\mathrm{Br} / \mathrm{Cl})_{3}$ QDs. By doping small amounts of $\mathrm{Mn}^{2+}$ (molar ratio of $0.19 \%$ ), the PLQY of PQD films improved from $9 \%$ to $28 \%$, which originated from the passivation of trap states that reduce the nonradiative decay. Benefiting from this $\mathrm{Mn}^{2+}$-doping strategy, deep-blue PeLEDs (466 nm) showed a high 
EQE of $2.12 \%{ }^{64}$. Pan et al. synthesized $\mathrm{Ni}^{2+}$-doped PQDs based on the room-temperature supersaturated recrystallization synthetic approach. The PLQY of the $\mathrm{Ni}^{2+}$-doped blue ( $470 \mathrm{~nm}$ ) PQDs reached up to $89 \%$ and $41 \%$ in dispersion and solid film state, respectively, which is a three-fold improvement compared to undoped $\mathrm{CsPbCl}{ }_{0.99} \mathrm{Br}_{2.01}$ QDs (Fig. 4(b)). The results of TRPL decay measurement suggest that the exciton trap state in the PQDs was efficiently eliminated by introducing an optimal amount of $\mathrm{NiCl}_{2}$, while more $\mathrm{NiCl}_{2}$ would result in new defect sites due to the increased concentration of chloride ions. The best $\mathrm{Ni}^{2+}$-doped PeLEDs exhibit a maximum luminance of $612 \mathrm{~cd} / \mathrm{m}^{2}$ with an EQE of $2.4 \%{ }^{65}$. In addition to doping metal ions, Shi et al. provided a ligand-mediated $\mathrm{Cl}^{-}$passivation strategy during the purification process of PQDs. By adding excess amounts of short-chain ligand $2-\mathrm{BACl}$, the halogen vacancies at the surface of $\mathrm{CsPbBr}_{3}$ QDs were passivated effectively. When the ratio of $\left[\mathrm{Cl}^{-}\right]_{\text {added }} /[\mathrm{Br}]_{\text {initial }}$ was 0.3 , the PL peak blue-shifted to $461 \mathrm{~nm}$ with a PLQY of 52\% in dispersion. Semiquantitative X-ray diffraction (XRD) analysis suggested that the concentration of $\mathrm{Cl}^{-}$at the surface of PQDs is much higher than that inside grains, indicating that $\mathrm{Cl}^{-}$can passivate the defect sites at the surface. The 2-Ba organic cations can effectively replace the OA and OAm ligands and prevent the agglomeration caused by ligand loss during the film formation process. However, this $\mathrm{Cl}^{-}$passivation strategy is not perfect as PeLEDs receive a severe red-shift during the operating process, with maximum luminance and $\mathrm{EQE}$ of only $76 \mathrm{~cd} / \mathrm{m}^{2}$ and 0.3 , respectively ${ }^{66}$. Similar strategy was provided by Shao et al. by doping short-chain ligands $\mathrm{PEACl}$ into the $\mathrm{CsPbCl}_{3}$ precursor solutions. Consequently, the PLQY of PEACl passivated PQDs increased from $5.4 \%$ to $62.3 \%$ due to the decreased $\mathrm{Cl}$-vacancies defects. To obtain PeLEDs in blue region, anion exchanges process was precisely controlled by mixing proper volume of TBABr solution into PEACl-PQDs dispersion, with the PLQY of PQDs further increased to $80.2 \%$ at $470 \mathrm{~nm}$. PeLEDs based on this PEACl modified PQDs showed the maximum luminance of $620 \mathrm{~cd} / \mathrm{m}^{2}$ at $468 \mathrm{~nm}^{67}$. Besides the organic or inorganic halide dopants, pseudohalides can also act as passivation agents to diminish the defect density of blue PQDs. Zheng et al. added n-dodecyl-ammonium thiocyanate (DAT), a kind of nonpolar solvent-soluble pseudohalide, into the $\mathrm{CsPb}\left(\mathrm{Br}_{x} / \mathrm{Cl}_{1-x}\right)_{3}$ QD dispersion (470 $\left.\mathrm{nm}\right)$, and the PLQY of PQDs in dispersion reached almost $100 \%$. DFT calculation indicated that the $\mathrm{SCN}^{-}$group can effectively fill the $\mathrm{Cl}^{-}$vacancies, as shown in the schematic diagram (Fig. 4(c)). The mid-gap trap states were eliminated and the density of states near the VBM increased due to the filled $\mathrm{Cl}^{-}$vacancies. Moreover, this pseudohalide doping strategy prevents ion exchange during the post-treatment process, meaning the emission spectrum of PQDs can remain stable. As-prepared blue PeLEDs (471 nm) exhibited a high EQE of $6.2 \%$, which is one of the most efficient PeLEDs in the spectra range of $460 \mathrm{~nm}$ to 480 $\mathrm{nm}^{68}$. Besides pseudohalides, Chiba et al doped adamantane-1,3-diamine (ADDA), a kind of adamantane derivatives with rigid bidentate structure, into the $\mathrm{CsPb}\left(\mathrm{Br}_{x} / \mathrm{Cl}_{1-x}\right)_{3} \mathrm{QD}$ dispersion to substitute the longchain ligand of OAm. The PL peak of PQDs remain unchanged at $456 \mathrm{~nm}$ after the ligand exchange process of ADDA, with the PLQY of PQD films increased from $11.6 \%$ to $31.8 \%$. The authors attributed this improvement to the suppressed non-radiative recombination by the amino-group of ADDA ${ }^{69}$. Wang et al. adopted hotinjection methods and synthesized $\mathrm{Pb}$-free deep-blue (445 nm) $\mathrm{Cs}_{3} \mathrm{Cu}_{2} \mathrm{I}_{5}$ QDs with a PLQY of up to $87 \%$. PeLEDs based on these $\mathrm{Cs}_{3} \mathrm{Cu}_{2} \mathrm{I}_{5}$ QDs exhibited a maximum EQE of $1.12 \%$, with excellent operational lifetime $\mathrm{T}_{50}$ of $170 \mathrm{~h}$ and stable EL spectra during the aging process (Fig. 4(d)). The device stability was further verified by an aggressive thermal cycling test (300-360-300 K) and a 35 -day storage test ${ }^{70}$.

As discussed above, the PLQY of PQDs in dispersion has reached $100 \%$ across the entire blue emission spectra region. However, the EQE of PQD-based devices is much lower than the PLQY in dispersion, which can be referred to the poor morphology and conductivity of PQDs films. To improve the quality of $P Q D s$ films, strategies are raised up in the following aspects: 1) Eliminating the long-chain ligands like OA and OAm during the preparation of PQDs. Short-chain organic ligands or metal ions can act as the capping agents to substitute the insulated long-chain ligands, which can not only facilitate the charge carriers injection into the PQDs, but also improve the ligand loss phenomenon since the electrostatic force between the short chain ligands/metal ions and the PQDs is strengthen. 2) Reducing the diameter of PQDs. PQDs with small diameter tends to form a compact alignment during the films formation process, which is beneficial for not only the charge transport among PQDs but also the coverage of PQDs films. As the ligand substitution has been proved to be an effective method to 

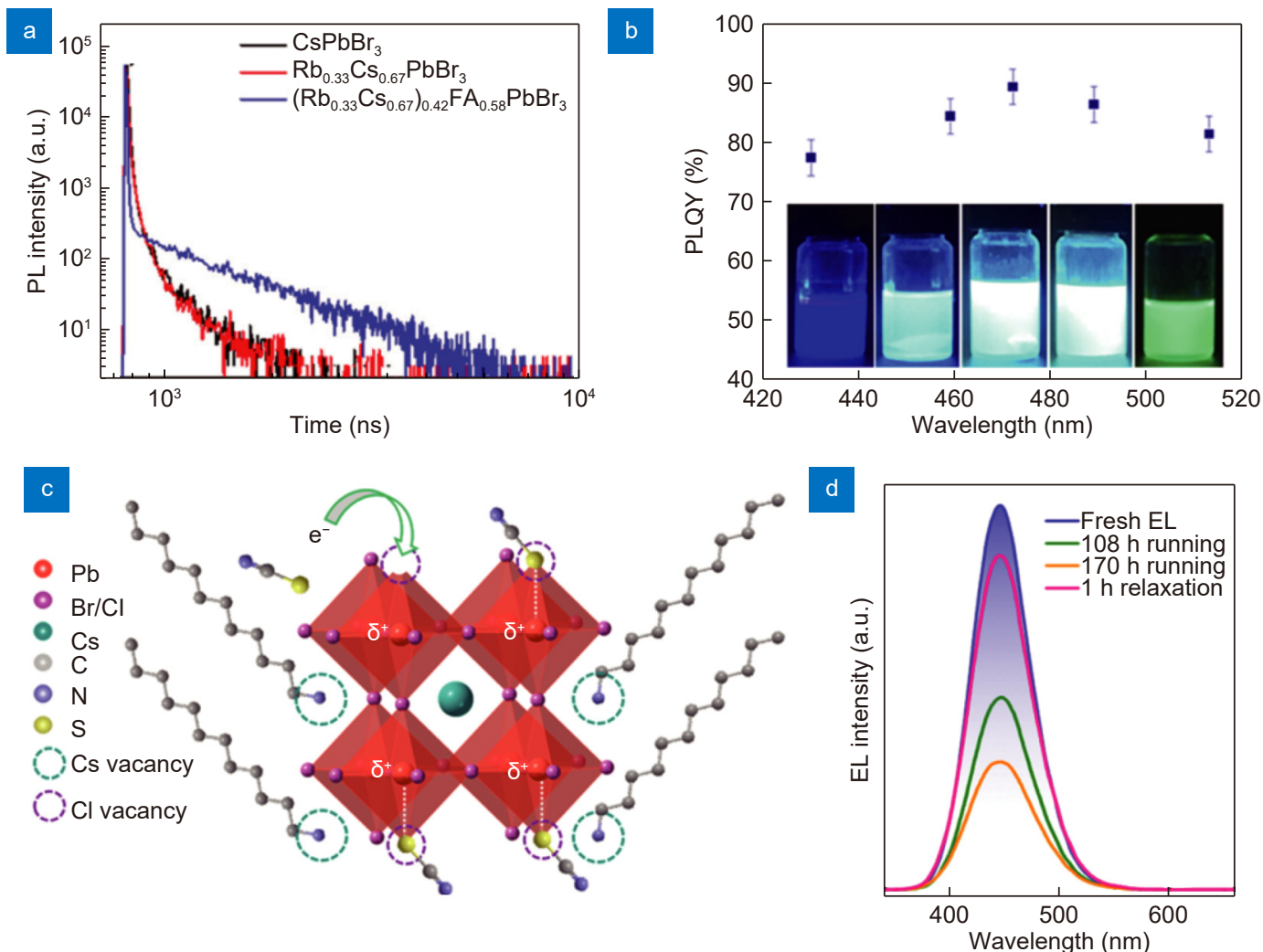

Fig. 4 | Defects passivation strategies for PQDs and their blue PeLEDs. (a) TRPL curves of pristine, RbBr-modified, and FABr/RbBr-modified PQD films. (b) PLQY of $\mathrm{Ni}^{2+}$ ion-doped $\mathrm{CsPbCl}_{x} \mathrm{Br}_{3-x} \mathrm{PQDs}$ in dispersion with $\mathrm{NiCl}_{2}$ precursor feeding amounts of $0,0.01,0.02,0.04$, and $0.08 \mathrm{ml}$. The inset shows photographs of the $\mathrm{Ni}^{2+}$ ion-doped $\mathrm{CsPbCl}_{x} \mathrm{Br}_{3-x} \mathrm{PQDs}$ under $365 \mathrm{~nm}$ UV lamp illumination. (c) Illustration of $\mathrm{Cl}$ va-

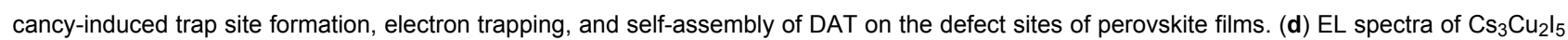
QD-based PeLEDs measured before aging, after running for 108 and $170 \mathrm{~h}$, and after a relaxation time of $1 \mathrm{~h}$. Figure reproduced with permission from: (a) ref..$^{63}$, Royal Society of Chemistry; (b) ref..$^{65}$, American Chemical Society; (c) ref. ${ }^{68}$, American Chemical Society; (d) ref. ${ }^{70}$, American Chemical Society.

decrease the diameter of PQDs, the category of the short chain ligands or metal ions selected for the substitution and the reaction condition should be thoroughly investigated.

\section{Colloidal perovskite nanoplatelets (PNLs)}

Besides the $\mathrm{Br} / \mathrm{Cl}$ mixing strategy, the bandgap of perovskites can also be enlarged by minimizing the grain size so it is smaller than the exciton Bohr radius to trigger the quantum confinement effect. Theoretical calculation exhibited that the exciton Bohr radii of $\mathrm{FAPbBr}_{3}$ and $\mathrm{CsPbBr}_{3}$ are $3.5 \mathrm{~nm}$ and $7 \mathrm{~nm}^{71}$, respectively, while most PQDs show a common radius of $\sim 10 \mathrm{~nm}$ with difficulty in further decreasing the particle size. Colloidal perovskite nanoplatelets (PNLs) are defined as perovskites synthesized by the colloidal method with much fewer layers of $[\mathrm{PbX} 6]^{4-}$ octahedra in one direction than in the other two directions. The lateral size can be precisely tuned from $1 \mathrm{~nm}$ to $4 \mathrm{~nm}$, which is small enough to trigger the quantum confinement effect, so the emitting spectrum can be adjusted in a wide range. Based on this quantum confinement effect, the composition of blue-emitting colloidal PNLs can be pure $\mathrm{Br}$, which is believed to exhibit a superior spectral stability compared with pure $3 \mathrm{D}$ perovskites and $\mathrm{PQDs}$ with $\mathrm{Br} / \mathrm{Cl}$ mixing composition. Moreover, $E_{\mathrm{B}}$ can be achieved at several hundred $\mathrm{meV}$, which is much higher than that of pure 3D perovskites and PQDs. Such a tightly bound exciton can promote the thermal stability of colloidal PNL-based PeLEDs, preventing the exciton quenching process caused by the high Joule heat produced during the operating process. Therefore, colloidal PNLs seem to be a promising blue-emitting perovskite material.

In 2015, Bekenstein et al. synthesized colloidal $\mathrm{CsPb}(\mathrm{Br} / \mathrm{Cl})_{3}$ PNLs for the first time by a similar hot-injection synthesis procedure as that for PQDs, with the reaction temperature decreased from $130{ }^{\circ} \mathrm{C}$ to $90{ }^{\circ} \mathrm{C}$. Ethyl acetate and methyl-ethyl ketone were selected to purify 
the PNLs without causing damage to the crystal structure. Colloidal PNLs with lateral sizes of $n=1$ to 5 (where $n$ refers to the number of $[\mathrm{PbX} 6]^{4-}$ octahedron layers) were synthesized simultaneously when the reaction temperature was $130{ }^{\circ} \mathrm{C}^{72}$. Akkerman et al. synthesized pure $\mathrm{Br}$ colloidal $\mathrm{CsPbBr}_{3}$ nanoplates for the first time. By decreasing the reaction temperature as well as adjusting the concentration of $\mathrm{HBr}$ in the precursor solution, the thickness of colloidal PNLs could be precisely tuned from 3 to 5 layers per unit cell ${ }^{73}$. For the fabrication of colloidal PNL-based PeLEDs, the grain size of PNLs, including the layers thickness and lateral length, must be precisely controlled to obtained the required emission spectra. Moreover, the requirement of the surface passivation is higher than that for pure $3 \mathrm{D}$ perovskites and PQDs because the amounts of surface defects of PNLs are much higher due to the high surface-to-volume ratio of PNLs.

\section{Grain size modification strategies}

The categories, concentrations of precursors, and reaction conditions greatly influence the grain size of colloidal PNLs and the morphology of PNL films, which can further influence device performance. In 2016, Kumar et al. synthesized colloidal $\mathrm{CsPbBr}_{3}$ PNLs with exact layer thicknesses of $n=1$ (deep-blue), $n=3$ (blue), and $n=5$ (sky-blue) through precise control of the concentrations and molar ratios of $\mathrm{OA}, \mathrm{OAm}$, and $\mathrm{MA}^{+}$. A room-temperature EL PeLED based on colloidal $\mathrm{CsPbBr}_{3}$ nanoplatelets as the emissive layer was fabricated for the first time (Fig. 5(a)). However, the maximum luminance and EQE degraded as the layers of PNLs decreased, which originated from the severe loss of ligands during the film formation process ${ }^{74}$. Todorovic et al. doped $\mathrm{Rb}^{+}$into the precursor solution and synthesized Rb-based colloidal PNLs for the first time by increasing the ratio of OA:OAm from 1:1 to 2:1. The as-synthesized colloidal PNLs show a deep-blue emission with a PL peak wavelength at $467 \mathrm{~nm}$. However, the PeLEDs based on the Rb-based colloidal PNLs show poor device performance, with a maximum luminance and EQE of $71 \mathrm{~cd} / \mathrm{m}^{2}$ and $0.11 \%$, respectively ${ }^{56}$. The loss of ligands during the purification process is also regarded as the main cause for the poor device performance. The above two results demonstrate the feasibility of exactly controlling the layer thickness of colloidal PNLs by adjusting the categories and concentrations of precursors, especially the ligands. However, the ligand loss phenomenon increases the difficulty of controlling the grain size of colloidal PNLs due to the weakened dispersity of colloidal PNLs, which can cause severe agglomeration of colloidal PNLs in solid film state. To minimize the degree of ligand loss, Zhang et al. adopted the post-treatment strategy by using $\mathrm{DDAB}$ as the capping ligands to strengthen the interaction with the surface of $\mathrm{CsPbr}_{3}$ PNLs, as shown in the schematic diagram (Fig. 5(b)). XRD measurement exhibited an enhanced intensity of the (100) and (200) peaks, indicating increased quantities of colloidal PNLs. The fourier-transform infrared spectroscopy (FTIR) indicated that DDA groups substitute the long-chain ligands of OAm at the surface of PNLs successfully. This is inspired by the fact that the valance band maximum of DDAB-treated colloidal PNLs shows an up-shift from 7.1 $\mathrm{eV}$ to $6.8 \mathrm{eV}$, further improving the hole injection into the colloidal PNLs. The DDAB-treated deep-blue (469 $\mathrm{nm})$ PeLEDs show a high EQE of $1.42 \%$, which is one of the most efficient colloidal PNL-based light emitting diodes $^{75}$. Besides adjusting the ligands, the layers of colloidal PNLs can be precisely controlled from $n=2$ to 6 by adjusting the ratios of $\mathrm{Cs} / \mathrm{Pb}$ in the precursor solution $^{76}$. As for the reaction conditions, Yang et al. studied the influence of initial temperature, heating rate, and reaction time on the grain size of colloidal PNLs. The results showed that the layers thickness increased as the reaction temperature and heating rate increased, with the lateral length remaining unchanged. This is because the reaction temperature significantly influences the interaction between ligands and the colloidal PNL surface. The lateral length of colloidal PNLs increased with layer thickness remaining unchanged by simply prolonging the reaction times. The enlarged lateral length originated from the Ostwald ripening process, which would not change the thickness of colloidal PNLs. However, the asformed colloidal PNL-based devices show a poor luminance of $25 \mathrm{~cd} / \mathrm{m}^{2}$ with the peak wavelength of the EL spectra red-shifted to $480 \mathrm{~nm}$. The stacking of the colloidal PNLs during the film formation process is the main reason for the red-shift phenomenon, while the poor device performance originates from the severe ligand loss ${ }^{77}$.

\section{Defect passivation strategies}

The defect passivation of colloidal PNLs mainly refers to the passivation of halogen vacancies defects at the surface of colloidal PNLs. Bohn et al. adopted the post-treatment strategy of blending $\mathrm{PbBr}_{2}, \mathrm{OA}$, and $\mathrm{OAm}$ in 
solution with a colloidal PNL dispersion with layer thicknesses of $n=2$ and 3. The increased concentration of $\mathrm{Br}^{-}$ in dispersion can effectively repair the $\mathrm{Br}$ vacancies at the surface of colloidal PNLs, with PLQY in dispersion increasing from $7 \%$ and $9 \%$ to $49 \%$ and $60 \%$ for colloidal PNLs with layer thicknesses of $n=2$ and 3, respectively. Deep-blue (464 nm) PeLEDs with colloidal PNLs $(n=3)$ as the emissive layer were fabricated with increased color purity (full width at half maximum (FWHM) $<20 \mathrm{~nm}$ ) (Fig. 5(c) $)^{76}$. A similar strategy was proposed by $\mathrm{Wu}$ et al. They added excess amounts of $\mathrm{PbBr}_{2}$ into the precursor solution and in-situ repaired the Br vacancies at the surface during the synthesis process of colloidal NPLs by increasing the concentration of $\mathrm{Br}^{-}$ions in solution, as shown in the schematic diagram (Fig. 5(d)). The additional $\mathrm{HBr}$ in the precursor solution facilitated dislocation between $\mathrm{Pb}^{2+}$ and the ligands and promoted the combination of $\mathrm{Pb}^{2+}$ and $\mathrm{Br}^{-}$. This $\mathrm{PbBr}_{2}$ in-situ treatment improved the PLQY of colloidal PNLs in dispersion from $18 \%$ to $96 \%$, with the highest PL color purity $(\mathrm{FWHM}=12 \mathrm{~nm})$. It is important to note that the
$\mathrm{PbBr}_{2}$-treated colloidal PNLs show great UV stability, with unchanged PL spectrum after stimulation by 405 $\mathrm{nm}$ UV lamps for $400 \mathrm{~min}$. Accordingly, the EL spectra of PeLEDs is overlapped with the PL spectrum with their maximum luminance and EQE at $62 \mathrm{~cd} / \mathrm{m}^{2}$ and $0.124 \%$, respectively. The oily and awful emitting film is the main reason for the relatively poor device performance ${ }^{78}$. Besides the halogen vacancies defects, the metallic $\mathrm{Pb}$ or $\mathrm{Pb}$ clusters in PNLs dispersion can form a nonradiative recombination channel that decrease the PLQY of the PNLs in dispersion or solid film state. To solve this problem, Peng et al. showed a trioctylphosphine oxide (TOPO)-mediated one-step modification strategy. The introduction of TOPO in precursor solution can eliminate the metallic $\mathrm{Pb}$ or $\mathrm{Pb}$ clusters by forming TOPO-Pb$\mathrm{Br}_{2}$ adduct. Moreover, the TOPO- $\mathrm{PbBr}_{2}$ adduct can served as the intermediate facilitating the deprotonation process of OA and OAm. As the result, the ligand concentration at the surface of TOPO-modified PNLS was increased, resulting in a reduced surface defects density. By this strategy, deep blue (439 nm) PeLED based on a

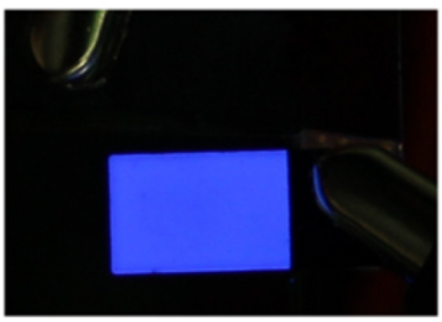

c

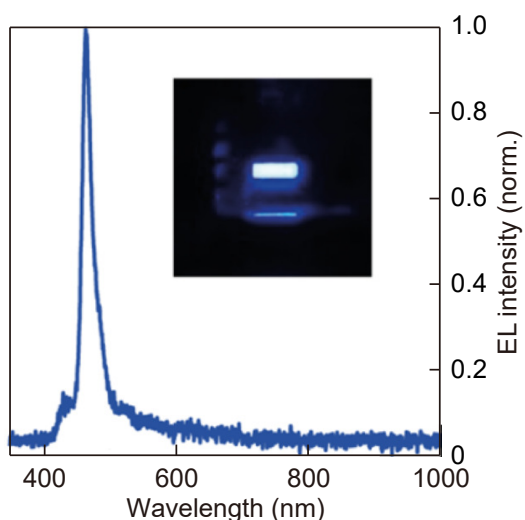

b

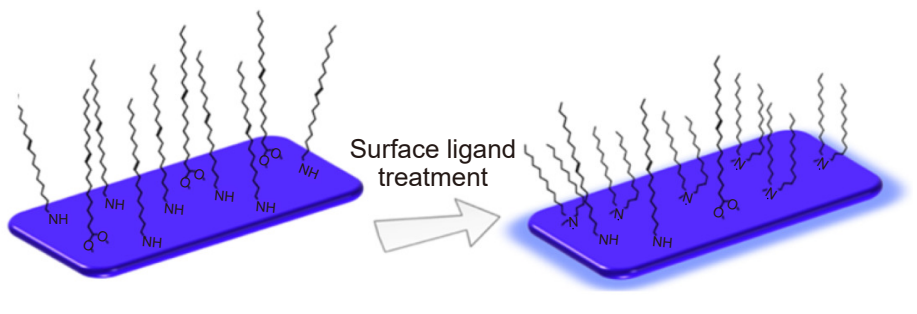

d
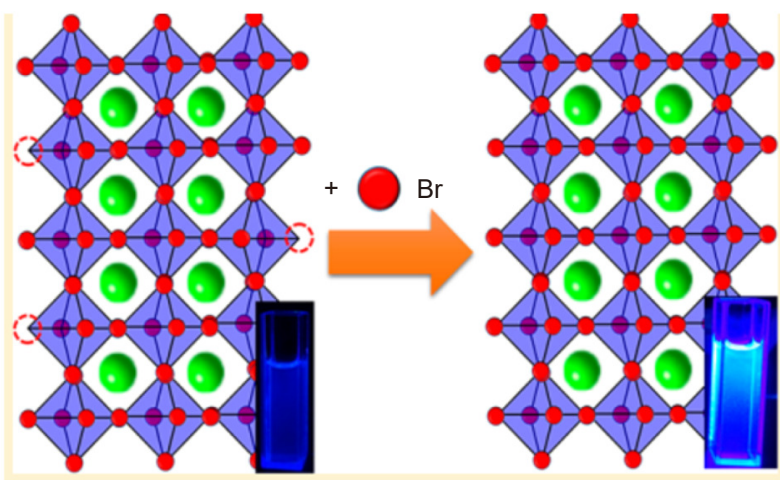

Fig. 5 | Dimension tuning and surface passivation strategies for PNLs and their blue PeLEDs. (a) A digital photograph of the first colloidal PNL-based pure-blue LED in operation (area: $3 \mathrm{~mm} \times 5 \mathrm{~mm}$ ). (b) Schematic diagram of colloidal NPLs treated by DDAB. (c) EL spectra of the colloidal PNL-based PeLEDs fabricated by Bohn et al. with $\mathrm{PbBr}_{2}$ post-treatment strategy. The inset shows digital photographs of a device in operation. (d) Illustration of in-situ passivation strategy of $\mathrm{PbBr}_{6}{ }^{4-}$ octahedra. Figure reproduced with permission from: (a) ref. ${ }^{74}$, American Chemical Society; (b) ref. ${ }^{75}$, American Chemical Society; (c) ref. ${ }^{76}$, American Chemical Society; (d) ref. ${ }^{78}$, American Chemical Society. 
$\mathrm{FAPbBr}_{3}$ nanoplatelets was achieved with the maximum $\mathrm{EQE}$ and FWHM of $0.14 \%$ and $14 \mathrm{~nm}$, respectively ${ }^{7 \%}$.

Colloidal PNLs are regarded as a promising candidate for PeLEDs with emission spectra in deep blue region. However, the largely existed defects at the surface of PNLs caused by the large surface/volume ratio facilitated the nonradiative recombination process. Those defects at the surface of PNLs can also strength the ligand loss phenomenon, which is harmful to the size control of PNLs due to the agglomeration of PNLs that can facilitate the unwanted Ostwald ripening process. Even though the post-treatment as well as in-situ passivation strategies can heal the defect sites at the surface of colloidal PNLs and increase the PLQY to near $100 \%$ in dispersion, Ostwald ripening process can happen during the preparation of PNLs films and device operation process so that degrading the device performance. To improve the device performance, surface defects as well as the Ostwald ripening process should be eliminated, which can be realized as the following aspects: 1) Eliminating the long-chain ligands like OA and OAm during the preparation of PNLs. This is the same strategy provided for PeLEDs based on PQDs films, since the short chain or metal ions can form a stronger affinity to the surface of PNLs, hindering the formation of surface defects. 2) Optimizing the film formation process. As the Ostwald ripening process can happen during the spin-coating and the annealing process, novel film formation strategy should be exploited to weaken the unwanted regrowth of PNLs.

\section{Quasi-2D perovskites}

Quasi-2D perovskites, also called Ruddlesden-Popper perovskites, are defined as perovskites with structure formula $\mathrm{L}_{2} \mathrm{~A}_{n-1} \mathrm{~B}_{n} \mathrm{X}_{3 n+1}$. The $\mathrm{L}$ site usually refers to bulk organic ammonium cations (abbreviated as bulk cations), the A site refers to the monovalent cations, the $\mathrm{B}$ site refers to the divalent cations, and the $\mathrm{X}$ site refers to halogens. The bulk cations can act as ligands in the colloidal synthesized PQDs and PNLs, which can limit the growth of perovskite grains. Quasi-2D perovskite films are composed of low-dimensional perovskites with limited $\left[\mathrm{PbX}_{6}\right]^{4-}$ octahedron layers $(n=1,2,3, \cdots$, where the definition of $n$ is analogous to that for colloidal PNLs) and high-dimensional perovskites $(n=\infty$ for $3 \mathrm{D}$ perovskites), which are formed simultaneously during the film formation process. This multi-phase structure can act as an energy ladder to promote energy transfer between the different phases. When applying quasi-2D perovskites as the emissive layers, the electron-hole pairs form in the lowest $n$ phases at first and then go through an energy funneling process, finally being collected in the highest $n$ phases. The high carrier concentration in the final perovskite emitting phases is beneficial for the radiative recombination process. Blue-emitting quasi-2D perovskites can be realized by adjusting the bandgap of the highest $n$ phases or lowering the dimension of the highest $n$ phases to trigger the quantum confinement effect.

It can be said that quasi-2D perovskites form a theoretical bridge between pure 3D and PNLs perovskites. Compared with pure 3D perovskites, quasi-2D perovskites exhibited an increased $E_{\mathrm{B}}$ due to the restricted grain size that can trigger the quantum confinement effect. Furthermore, the defect densities as well as water/oxygen stability are well-modified compared with those of 3D perovskites thanks to the passivation and protection effects of the bulk cations. Compared with colloidal PQDs and PNLs, quasi-2D perovskite films can be formed directly from a precursor solution without a purification process, so the ligands loss phenomenon can be fully eliminated during the film formation process. Moreover, the carbon chain length of commonly used bulk cations in quasi-2D perovskites is much shorter than that in long-chain ligands, such as OA and OAm, so the conductivity of quasi-2D perovskite films can be much better compared with that of PQD or PNL films.

In early studies, researchers aimed to find suitable bulk cations to fabricate blue-emitting quasi-2D PeLEDs. $\mathrm{Hu}$ et al. doped $\mathrm{BABr}$ into the precursor and fabricated RGB quasi-2D PeLEDs by the mixed halides strategy. However, the luminance and efficiency of sky-blue PeLEDs are considerably lower those of red and green ones because of the considerably high leakage current and poor charge injection into perovskite films ${ }^{80}$. Congreve et al. studied the effect of the concentration of BA cations on the composition of perovskite films. The perovskite films were fabricated by mixing the precursor stoichiometrically with target composition of $n=2$ phase. However, although the absorption spectrum confirmed that quasi-2D perovskites mainly has $n=2$ and 3 phases, the green PL emission of the obtained quasi-2D perovskite films indicated the formation of the $3 \mathrm{D} \mathrm{MAPb}-$ $\mathrm{Br}_{3}$ perovskite $(n=\infty)$. With the excess amount of BA cations, the grain growth was further restricted, and the blue-emitting quasi-2D perovskite film was fabricated 
with compositions of $n=2,3$, and 4 . However, the obtained blue PeLEDs showed an extremely low EQE of $0.0054 \%$ with a broad emission spectra, which originated from the poor current injection caused by an excess amount of BA cation $s^{81}$. In addition to BA cations, several bulk cations have been applied to fabricated blue-emitting quasi-2D PeLEDs. Cheng et al. doped 4-PBABr into the precursor solution, and sky-blue $(491 \mathrm{~nm})$ quasi-2D PeLEDs were fabricated with a maximum luminance of $186 \mathrm{~cd} / \mathrm{m}^{2}$. However, both PL and EL spectra were broad with multiple emission peaks, indicating poor energy transfer between quasi-2D perovskite phases due to the excess amount of insulating 4-PBA cations ${ }^{82}$. Chen et al. fabricated quasi-2D PeLEDs by selecting POEA as bulk cations. POEA can strongly react with $\mathrm{MAPbBr}_{3}$ and limit the direction of grain growth. The morphology of POEA-doped perovskite film changed from rough and discontinuous to continuous and rod-like shaped, finally achieving dense and uniform film morphology. By increasing the molar ratio of POEA cations, the PL and EL spectra blue-shifted from $530 \mathrm{~nm}$ to $462 \mathrm{~nm}$. When the molar ratios of POEA were $40 \%$ and $60 \%$, the corresponding sky-blue and blue PeLEDs exhibited maximum EQEs of $1.1 \%$ and $0.06 \%$, respectively. However, the same energy transfer problem still exists with broad PL and EL spectra when the concentration of POEA exceeds $30 \%{ }^{83}$. Wang et al. doped $\mathrm{EABr}$ into the $\mathrm{MAPbBr}_{3}$ precursor solution, and the same hypsochromic shift of both PL and EL spectra was observed when the concentration of $\mathrm{EABr}$ was increased. When the ratio of $\mathrm{EABr}$ to $\mathrm{MABr}$ was $1.3: 1$, the obtained blue-emitting quasi-2D PeLED exhibited a maximum luminance and EQE of $200 \mathrm{~cd} / \mathrm{m}^{2}$ and $2.6 \%$, respectively. However, the PL and EL spectra exhibited two emission peaks at $473 \mathrm{~nm}$ and $485 \mathrm{~nm}$, respectively due to the excess $\mathrm{EABr}^{84}$.

Previous studies have confirmed the significant potential of quasi-2D perovskites for the fabrication of blueemitting PeLEDs. However, the following two main problems must be resolved for further improvement of device performance. One of the problems is the modulation of the phase distribution. As quasi-2D perovskite films are composed of multiple phases, the energy funneling process limits deep-blue emission because the excitons or free charges will finally be concentrated and radiatively recombined in the phases with the narrowest bandgap (the highest $n$ phases). While adding excess amounts of bulk cations can facilitate the generation of low- $n$ phases and more effectively passivate surface de- fects, the insulating property of bulk cations can degrade the charge transfer efficiency between different phases, such that the color purity of quasi-2D blue PeLEDs is degraded with multiple emission peaks. In fact, the PLQY, conductivity, and color purity of quasi-2D perovskite films cannot be optimized simultaneously by simply adjusting the concentration of the bulk cations; therefore, strategies for the precise modulation of phase distribution should be exploited. The other problem is the stability of the EL spectra. Although most bulk cations are hydroscopic which can effectively improve the water stability of quasi-2D perovskites. Exposure to UV light, electric field, and Joule heat produced during the operating process can destabilize the quasi-2D perovskites and lead to the phase segregation phenomenon, which can cause the red-shift of emission spectra, decreasing the device operating lifetime. In this section, strategies for phase modulation as well as the improvement of spectral stability are reviewed.

\section{Phase modulation strategies}

Phase modulation strategies are performed to obtain blue-emitting quasi-2D perovskite films with narrow emission spectra, short emission wavelength, and high PLQY. The primary task for the phase modulation is to obtain blue-emitting perovskite films, which can be realized through either enlarging the bandgap of 3D phases in quasi-2D perovskite films or narrowing the phase distribution with only low- $n$ phases to trigger the quantum confinement effect. Generally, the 3D phases have the narrowest bandgap in quasi-2D films, which can be effectively enlarged by the mixed $\mathrm{Br} / \mathrm{Cl}$ strategy. Moreover, when applying the mixed $\mathrm{Br} / \mathrm{Cl}$ strategy, the concentration of bulk cations can be adjusted to a suitable range without reducing the conductivity. Vashishtha et al. prepared all inorganic quasi-2D blue PeLEDs for the first time by simultaneously adjusting the concentration of $\mathrm{BA}$ cations as well as the ratio of $\mathrm{Br} / \mathrm{Cl}$ in precursor solutions. The EL spectra exhibited a wide tuning range with EL peak wavelength varied from $465 \mathrm{~nm}$ to $500 \mathrm{~nm}$ (Fig. 6(a)). The concentration of BA cations was also well controlled to achieve PeLEDs with pure emission. The FWHM of the EL spectra were $22 \mathrm{~nm}$ and $23 \mathrm{~nm}$ for the sky-blue $(488 \mathrm{~nm})$ and deep-blue $(465 \mathrm{~nm})$ PeLEDs, respectively. The EL performance of the sky-blue and deep-blue PeLEDs was improved, with maximum luminances of $3340 \mathrm{~cd} / \mathrm{m}^{2}$ and $962 \mathrm{~cd} / \mathrm{m}^{2}$ and EQEs of $6.2 \%$ and $2.4 \%$, respectively ${ }^{85}$. A similar strategy was adopted 
by Wang et al. by finely modifying the molar ratio of PBA cations and $\mathrm{Cl}^{-}$. When the molar ratio of $\mathrm{Cl}^{-}$was fixed at $30 \%$, the PL spectra could be adjusted in the range from $496 \mathrm{~nm}$ to $472 \mathrm{~nm}$ by increasing the molar ratio of $\mathrm{PBA}$ cations from $10 \%$ to $100 \%$ with narrow FWHM $(21 \mathrm{~nm})$ of the PL spectra. When the molar ratio of PBA cations is $72 \%$, the PLQY of the as-prepared quasi-2D film reached a maximum of $42 \%{ }^{86}$. Li et al. clarified that it is impossible to eliminate the formation of 3D phases when using PEA as the sole bulk cation. For the fabrication of blue PeLEDs, they first controlled the emission peak position by increasing the molar ratio of $\mathrm{CsCl}$ to $\mathrm{CsBr}$; they reached the shortest peak wavelength at $484 \mathrm{~nm}$ when the molar ratio of $\mathrm{CsCl}$ to $\mathrm{CsBr}$ reached a maximum of 9:1. By further optimizing the concentration of PEA cations, the peak wavelength could be further blue-shifted to $480 \mathrm{~nm}$ with an improved PLQY of 27\% (Fig. 6(b)). For the further optimization of device performance, the thickness of PEDOT:PSS was decreased to obtain an optimal position of the radiative recombination center. The best sky-blue $(480 \mathrm{~nm})$ device showed a maximum luminance and EQE of $3780 \mathrm{~cd} / \mathrm{m}^{2}$ and $5.7 \%$, respectively, with a narrow EL FWHM of $21 \mathrm{~nm}^{87}$. In order to further improve the efficiency of quasi-2D blue PeLED based on $\mathrm{Br} / \mathrm{Cl}$ mixing strategy, Zhang et al. doped $\mathrm{GaBr}$ into perovskite precursor and successfully fabricated perovskite films with efficient 2D/3D energy cascaded channels via a simple one-step spin-coating method. By precisely controlling the molar ratio of $\mathrm{GaBr}$, the electronic energy band structure was reconstructed, which is benefit for the transport of electron-hole pairs from $2 \mathrm{D}$ phases to the $3 \mathrm{D}$ phases. Also, the injected charge carriers can be effectively confined inside the 3D phases due to the dielectric property of $\mathrm{GaBr}$ bulk cations. By the synergistic effect of enhanced energy funneling efficiency and charge confinement, skyblue $(492 \mathrm{~nm})$ quasi-2D PeLEDs were fabricated with maximum $\mathrm{EQE}$ of $8.2 \%{ }^{88}$. Besides the $\mathrm{Br} / \mathrm{Cl}$ mixing strategy, the bandgap of the $3 \mathrm{D}$ phases in the quasi-2D perovskite films can also be enlarged by doping $\mathrm{Rb}^{+}$into the precursor solution as $\mathrm{Rb}^{+}$incorporation into the perovskite structure can adjust the energy level of VBM and CBM through controlling the degree of octahedral titling. Jiang et al. doped $\mathrm{RbBr}$ into the $\mathrm{Cs} \mathrm{PbBr}_{3}$ precursor solution and fabricated Rb-Cs-alloyed quasi-2D deepblue PeLEDs for the first time. The stoichiometrically prepared $\mathrm{PEA}_{2}\left(\mathrm{Rb}_{0.6} \mathrm{Cs}_{0.4}\right)_{2} \mathrm{~Pb}_{3} \mathrm{Br}_{10}$ films were fabricated with peak wavelength in the deep-blue region of $466 \mathrm{~nm}$.
Although the emissive peak position of films with excess amounts of $\mathrm{RbBr}$ received a red-shift from $466 \mathrm{~nm}$ to 476 $\mathrm{nm}$ compared with the stoichiometrically prepared $\mathrm{Rb}$ Cs-alloyed films, the energy transfer process is smoother and faster thanks to the optimized phases distribution. By doping excess amounts of $\mathrm{RbBr}$, the PLQY of films reached a maximum of $82 \%$ (Fig. 6(c)). The best Rb-Csalloyed PeLED showed a maximum luminance of $100.6 \mathrm{~cd} / \mathrm{m}^{2}$ and EQE of $1.35 \%$, with the FWHM of the EL spectra approximately $20 \mathrm{~nm}^{89}$.

Besides enlarging the bandgap of 3D phases in quasi2D perovskite films, the other way to obtain blue-emitting quasi-2D-based PeLEDs is to narrow the phase distribution of the obtained quasi-2D perovskite films with low- $n$ phases to trigger the quantum confinement effect. The dimension of quasi-2D phases can be restricted with only low- $n$ phases $(n \leq 4)$ existing by precisely controlling the crystallization rate. Liu et al. optimized the crystallization rate by adding large amounts of PBA cations and using ethyl acetate as an anti-solvent to wash away excess PBA cation. The composition of the as-prepared quasi-2D perovskite films was unique with $\mathrm{Cs}_{x} \mathrm{Fa}_{1-x} \mathrm{~Pb}$ $\mathrm{Br}_{3}$ nanocrystals (NCs) embedded within the layered perovskites. The radius of $\mathrm{Cs}_{x} \mathrm{Fa}_{1-x} \mathrm{PbBr}_{3} \mathrm{NCs}$ can be adjusted from $5.3 \AA$ to $6.7 \AA$ by decreasing the molar ratio of PBA cations, which leads to an adjustable emission spectra from $474 \mathrm{~nm}$ to $494 \mathrm{~nm}$. The obtained films showed a high PLQY of $60 \%$ with a low stimulation power of $0.24 \mathrm{~mW} / \mathrm{cm}^{2}$, indicating nearly no energy loss during the energy transfer process. The as-prepared PeLEDs showed a maximum luminance and EQE of $700 \mathrm{~cd} / \mathrm{m}^{2}$ and $9.5 \%$, respectively ${ }^{90}$. Xing et al. discovered that the strong Van der Waal's interaction between PEA cations promotes the crystallization rate of $n=1$ phases. The largely produced $n=1$ phases consume excess PEA cations and lead to the formation of $n=\infty$ phases $^{91}$. By partially replacing the PEA cations with short-chain iso-propylammonium (IPA) cations, the crystallization rate of $n=1$ phase dropped because of the weakened interaction between PEA cations. The main composition of the quasi-2D perovskite films also changed from both $n=1$ and $n=\infty$ to $n=2,3$, which is confirmed by the transient absorption measurement (Fig. 6(d)). By further optimizing the molar ratios of IPA, MA, and Cs, the PL peak wavelength blue-shifted from $504 \mathrm{~nm}$ to $467 \mathrm{~nm}$, with a high PLQY of $88 \%$ at a peak wavelength of $490 \mathrm{~nm}$. The sky-blue $(490 \mathrm{~nm})$ quasi-2D PeLED exhibited a high luminance of 
a

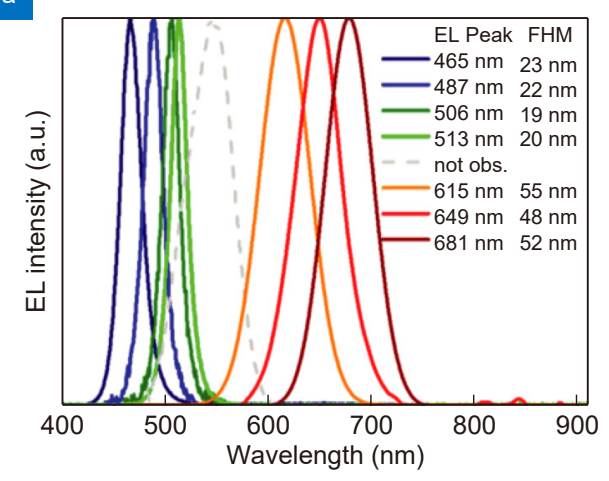

c

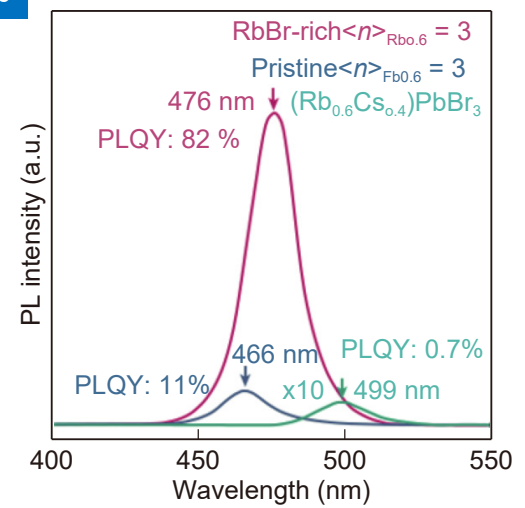

b

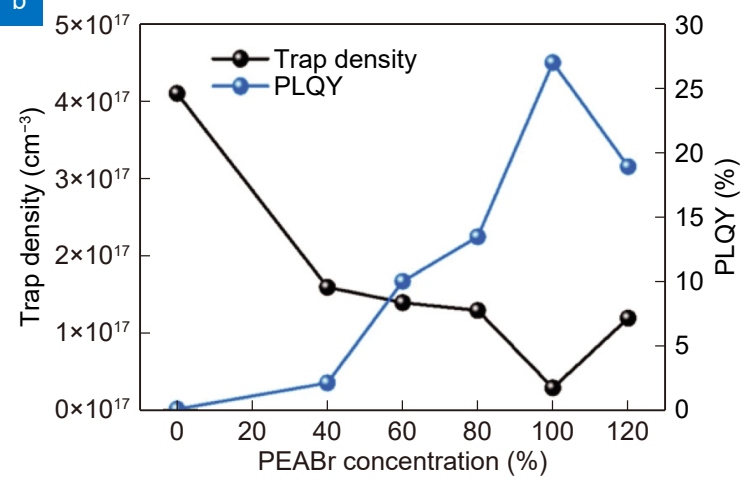

d

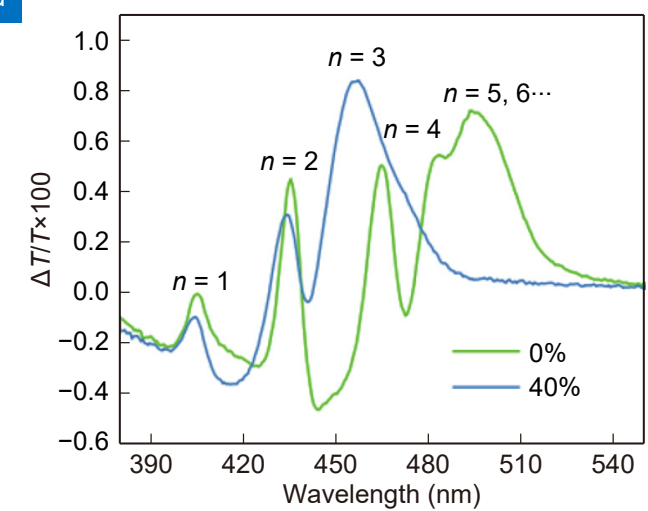

Fig. 6 | Phases modulation strategies for quasi-2D perovskite and their PeLEDs. (a) EL spectra of BA cations-based quasi-2D PeLEDs. (b) PLQY and trap density curves of quasi-2D perovskite film with various concentration of PEABr. (c) PL spectra of Rb-Cs alloyed perovskite films with various composition. (d) Transient absorption spectra of $\mathrm{PEA}_{2} \mathrm{MA}_{1.5} \mathrm{~Pb}_{2.5} \mathrm{Br}_{8.5}$ with various molar ratio of $\mathrm{IPABr}$ from 0 to $40 \%$. Figure reproduced with permission from: (a) ref. ${ }^{85}$, American Chemical Society; (b) ref. ${ }^{87}$, Springer Nature; (c) ref. ${ }^{89}$, Springer Nature; (d) ref. ${ }^{91}$, Springer Nature.

$2480 \mathrm{~cd} / \mathrm{m}^{2}$. Yuan et al. synthesized bi-amino bulk organic halide $\mathrm{P}-\mathrm{PDABr}_{2}$ and fabricated deep-blue $(465 \mathrm{~nm})$ quasi-2D PeLEDs with a high EQE of $2.6 \%$. The P-PDA cations possess two amino groups that can promote the crystallization of $n=2$ and $n=3$ phase due to the stronger interaction between P-PDA cations and $\left[\mathrm{PbBr}_{6}\right]^{4-}$ cages than between PEA and BA cations with only one amino group. The phase distribution can be further optimized by adding dual cations of $\mathrm{P}-\mathrm{PDABr}_{2}$ and PEA simultaneously. When the ratio of P-PDA: PEA is 3:1, the perovskite films show a narrow phase distribution with only $n=2$ and $n=3$ phases existing. The optimal quasi-2D perovskites showed a high PLQY of $77 \%$ at a PL peak wavelength of $465 \mathrm{~nm}$, indicating a high energy transfer efficiency ${ }^{92}$. Similar bi-amino cations were presented by He et al. to modify the compositions of quasi-2D perovskites. The bi-amino cation $\mathrm{BDADBr}_{2}$ was added to the $\mathrm{MAPbBr}_{3}$ precursor, and the PL emission peaks of the obtained quasi-2D perovskites shifted from $517 \mathrm{~nm}$ to $494 \mathrm{~nm}$ when the molar ratio of
$\mathrm{MABr}: \mathrm{BDBABr}_{2}$ changed from 8:1 to 2:1. Moreover, the PLQY of films further improved from $24.1 \%$ to $40.2 \%$ by further doping a small amount of PEO into the precursor solutions. With these modification strategies, the EQE of the obtained quasi-2D sky-blue PeLED increased from $1 \%$ to $2.7 \%{ }^{93}$.

Several novel film formation strategies have been applied to fabricate quasi-2D perovskite films with pure phase, which is beneficial for the precise control of the emission spectra. Liang et al. fabricated pure 2D $\mathrm{PEA}_{2} \mathrm{PbBr}_{4}$ perovskites using DMF vapor annealing methods. Deep-blue $(410 \mathrm{~nm})$ PeLEDs based on $\mathrm{PEA}_{2} \mathrm{PbBr}_{4}$ nanoplates as the emissive layer were fabricated for the first time. The obtained $\mathrm{PEA}_{2} \mathrm{PbBr}_{4}$ nanoplates fabricated by the DMF vapor annealing methods showed enlarged lateral length on micrometer size, which could reduce the defect density and enhance the PLQY due to the diminished grain boundaries. However, the discontinuous film morphology caused large leakage current, with the optimal device exhibiting a low EQE of 
$0.02 \%{ }^{94}$. To improve the morphology of $\mathrm{PEA}_{2} \mathrm{PbBr}_{4}{ }^{-}$ based perovskite films, Deng et al. adopted a novel film formation method called electric-field-deposition (EFD). Dense and uniform $\mathrm{PEA}_{2} \mathrm{PbBr}_{4}$ films with PL peak wavelength at $408 \mathrm{~nm}$ were fabricated with extremely narrow EL spectra $(F W H M=11.6 \mathrm{~nm})$. The PLQYs of the obtained $\mathrm{PEA}_{2} \mathrm{PbBr}_{4}$ films were in range of 15 to $25 \%$. $\mathrm{PEA}_{2} \mathrm{PbBr}_{4}$ films fabricated by the EFD method exhibited good water stability, with PL intensity maintained at approximately $65 \%$ in a moist environment with a relative humidity of $60 \%$ for 13 days. PeLEDs based on $\mathrm{PEA}_{2} \mathrm{PbBr}_{4}$ films fabricated by the EFD method exhibited a maximum luminance and EQE of $147.6 \mathrm{~cd} / \mathrm{m}^{2}$ and $0.31 \%$, respectively. The operating lifetime also improved significantly, with the initial luminance maintained at approximately $80 \%$ after continuously operating for $23 \mathrm{~h}$ at $5 \mathrm{~V}^{95}$. Chen et al. prepared Ruddlesden-Popper phase single crystals and fabricated PeLEDs based on the corresponding single crystals for the first time. The emissive layer based on $(\mathrm{BA})_{2} \mathrm{Cs}_{n-1} \mathrm{~Pb}_{n^{-}}$ $\mathrm{Br}_{3 n+1}$ single crystals was fabricated via a micromechanical exfoliation approach. (BA) ${ }_{2} \mathrm{Cs}_{n-1} \mathrm{~Pb}_{n} \mathrm{Br}_{3 n+1}$ single crystals with dimensions of $n=1,2$, and 3 were prepared and PeLEDs based on the $n=2$ and $n=3$ phases showed maximum EQEs of $0.7 \%$ and $1.1 \%$ with EL peak wavelengths of $450 \mathrm{~nm}$ and $482 \mathrm{~nm}$, respectively. The relatively low EQEs of these single crystal-based PeLEDs originated from the discontinuous film morphology, which caused a large leakage current with a short operating lifetime of only $10 \mathrm{~s}$ at $6 \mathrm{~V}$. Further investigation exhibited that the generated Joule heat during the operating process caused a quick spike in device temperature to $450 \mathrm{~K}$, which is the main cause of the poor device stability ${ }^{96}$.

\section{Spectra-stabilizing strategies}

Spectral stability refers to the ability of the emission spectra to remain stable against harsh environmental conditions, such as UV exposure, water/oxygen exposure, high temperature, and high operating voltage. Due to the large difference of the electronegativity between $\mathrm{Br}^{-}$and $\mathrm{Cl}^{-}$, the $\mathrm{Br} / \mathrm{Cl}$ mixed PeLEDs showed severe phase separation during the device operating process, which caused red-shift of the EL spectra. For example, the PeLEDs fabricated by Vashishtha ${ }^{85}$ et al. showed severe red-shift of EL spectra when the operating voltage surpassed $8.5 \mathrm{~V}$. Wang et al. fabricated sky-blue PeLEDs with a molar ratio of $\mathrm{Cl}^{-}$of $30 \%$, which showed a redshift of the EL spectra from $490 \mathrm{~nm}$ to $495 \mathrm{~nm}$ after op- erating for 6 min at $9 \mathrm{~V}$. They also found that the degree of red-shift was more severe when further increasing the molar ratio of $\mathrm{Cl}^{-86}$. The same result was also reported by Li et al., whose PeLEDs suffered a red-shift of the EL spectra from $480 \mathrm{~nm}$ to $488 \mathrm{~nm}$ by either increasing the operating voltage above $6 \mathrm{~V}$ or prolonging the operating time beyond $9 \min ^{87}$.

Several strategies have been provided to improve the EL spectral stability of PeLEDs based on $\mathrm{Br} / \mathrm{Cl}$ mixed phases. Tan et al. found that the red-shift of EL spectra during operation is reversible, indicating that the phase separation process caused by the electric fields is recoverable. When the applied voltage was changed from direct voltage to square-wave alternating voltage (SWAV), this spectrum recovering process could be sped up. By optimizing the voltage bias ( $6 \mathrm{~V}$ for forward direction and $4 \mathrm{~V}$ for reverse direction), duty ratio (0.25), and frequency $(50 \mathrm{~Hz})$ of the SWAV, the EL peak position could remain unchanged at $466 \mathrm{~nm}$ for $12 \mathrm{~h}$, which is one of the blue PeLEDs with the most stable EL spectra ${ }^{97}$. Wang et al. improved the EL spectral stability by doping both $\mathrm{PEACl}$ and $\mathrm{YCl}_{3}$ into quasi-2D perovskite films. The obtained sky-blue (487 nm) PeLEDs showed high EL spectral stability with the EL spectra remaining stable under continuous operation for $120 \mathrm{~min}$ at $3.2 \mathrm{~V}$. The results showed that the active energy of the ion migration increased with the incorporation of $\mathrm{PEACl}$ and $\mathrm{YCl}_{3} . \mathrm{YCl}_{3}$ was also proved to form a passivation layer outside the perovskite grains, which benefits from the passivation of halogen vacancies and further decreases halogen migration, as shown in the schematic diagram (Fig. 7(a)). The maximum luminance and $\mathrm{EQE}$ of the sky-blue $(487 \mathrm{~nm})$ quasi-2D PeLEDs were $9048 \mathrm{~cd} / \mathrm{m}^{2}$ and $11 \%$, respectively, which is one of the most efficient blue PeLEDs ${ }^{98}$. $\mathrm{Ma}$ et al. adopted the chloride insertion-immobilization strategy by doping the organic halide diphenylphosphinic chloride (DPPOCl) into the precursor solution. DFT calculation as well as ${ }^{31} \mathrm{P}$ NMR measurement suggested that DPPOCl can react with a trace amount of water in DMSO solution, and the as-formed DPPOOH can form phosphoryl bonds with the unsaturated $\mathrm{Pb}$ and halogen ions. By this strategy, the $\mathrm{Cl}^{-}$in the quasi-2D perovskites was in-situ immobilized and the emission spectral stability was greatly improved. The quasi-2 $\mathrm{D} \mathrm{PEA}_{2} \mathrm{Cs}_{1.6} \mathrm{MA}_{0.4^{-}}$ $\mathrm{Pb}_{3} \mathrm{Br}_{10}$ perovskite films treated by $30 \mathrm{mg} / \mathrm{ml} \mathrm{DPPOCl}$ showed a high PLQY of $54 \%$ with significantly improved thermal and UV stability, while the PeLEDs modified with $30 \mathrm{mg} / \mathrm{ml}$ DPPOCl showed peak EL position at 489 
$\mathrm{nm}$ with a narrow EL FWHM of $18 \mathrm{~nm}$. The operating lifetime $\mathrm{T}_{50}$ was $51 \mathrm{~min}$ for an initial luminance of $1500 \mathrm{~cd} / \mathrm{m}^{2}$ with the unchanged EL spectra during the operating process (Fig. 7(b) $)^{99}$. Wang et al. adopted a dual-ligands strategy to improve the EL spectra stability and operational lifetime of PeLED with $\mathrm{Br} / \mathrm{Cl}$ mixed phases. Two bulk cations of i-butylammonium (iBA) and phenylethylammonium were selected as capping ligands. The iBA cations has a large binding energy that can effectively passivate the defect sites of perovskite and weaken the halogen migration. By optimizing the molar ratio of the two bulk cations, sky-blue $(485 \mathrm{~nm})$ quasi-2D PeLEDs with maximum EQE of $7.84 \%$ was achieved. The operating lifetime $\mathrm{T}_{50}$ was $311 \mathrm{~min}$ for an initial luminance of $100 \mathrm{~cd} / \mathrm{m}^{2}$ with the EL spectra remain stable during the continuous operation for $300 \mathrm{~s}^{100}$.

Usually, pure Br-based PeLEDs show better spectral stability due to the relieved phase segregation effect. However, red-shift of emission spectra can still occur for pure Br-based PeLEDs due to the regrowth of quasi-2D perovskites through the Ostwald ripening process. Leung et al. adopted DFT calculations and found that compared with sole BA cation-based quasi-2D perovskites, quasi-2D perovskites with BA/PEA mixing showed better thermal stability due to minimized crystalline strain relaxation during the heating process. They also observed that the EL spectra of the device based on sole BA cations was red-shifted compared with the PL spectra after the thermal evaporation process, while the EL peak position of the device based on PEA/BA mixing remained stable at $456 \mathrm{~nm}$. The temperature of perovskite films can rise up to $95{ }^{\circ} \mathrm{C}$ during the thermal evaporation process, which leads to the regrowth of quasi-2D perovskites through the Ostwald ripening process, causing red-shift of the EL spectra ${ }^{101}$. The BA/PEA mixing strategy is regarded as an effective way to promote the thermal stability of quasi-2D perovskites. Besides thermal evaporation, the Joule heat produced during the device operating process is the main reason for the Ostwald ripening process. To decrease the Joule heat, nonradiative recombination as well as leakage currents should be diminished simultaneously, which can be realized through the effective passivation of defect sites and the modification of film morphology. Zeng et al. doped short-chain ligand DMA into a quasi-2D perovskite precursor containing either BA or PEA cations to reduce the defect density of the quasi-2D perovskite films. When the molar ratios of DMABr:PEABr and DMABr:BABr were 0.8 and 0.4 , respectively, the blue-greenish $(499 \mathrm{~nm})$ and sky-blue (490 nm) perovskite films showed PLQYs of $63.29 \%$ and $52.8 \%$, respectively. The improved PLQY indicates that the DMA cations can effectively passivate the defect sites in quasi-2D perovskites. The EL spectral stability was improved with unchanged EL peak position when the applied voltage shifted from $5 \mathrm{~V}$ to $13 \mathrm{~V}^{102}$. Jin et al. improved the thermal and UV stability of pure Brbased quasi-2D perovskite films by mixing the bulk cations of $\mathrm{PEABr}$ and $\mathrm{NPABr}_{2}$ simultaneously ${ }^{103}$. The film morphology was dense and uniform with a surface roughness of only $0.98 \mathrm{~nm}$. The obtained films showed excellent spectral stability, with unshifted PL peak position during the thermal annealing at $70{ }^{\circ} \mathrm{C}$ for $100 \mathrm{~min}$ or UV exposure for $100 \mathrm{~min}$ with high-power UV lamps at $12 \mathrm{~W}$. Moreover, the device stability was improved with unchanged EL peak position under continuous operation for $400 \mathrm{~s}$ at $5 \mathrm{~V}$ and $\mathrm{T}_{50}$ of $4.3 \mathrm{~min}$ at an initial luminance of $100 \mathrm{~cd} / \mathrm{m}^{2}$. In addition to decreasing the Joule heat, the Ostwald ripening process can also be relieved by separating the quasi-2D perovskite grains with thermally stable phases. Zou et al. improved the spectral stability of quasi-2D perovskites based on a moisturetreated strategy. They found that excess amounts of $\mathrm{CsBr}$ can facilitate the formation of $\mathrm{Cs}_{4} \mathrm{PbBr}_{6}$ phases and lower the PLQY of quasi-2D perovskite films. However, $\mathrm{Cs}_{4} \mathrm{PbBr}_{6}$ can be dissolved partly through the post-treatment with suitable water, while the remaining $\mathrm{Cs}_{4} \mathrm{PbBr}_{6}$ can form a passivation layer outside the perovskite grains that can not only reduce the defect density but also separate the quasi-2D perovskites to prevent the thermallyinduced regrowth process, which helps in improving the stability of the EL spectra (Fig. 7(c)). However, the moisture-treated perovskite films showed poor film morphology, which caused severe leakage current and degraded the devices' operating lifetimes ${ }^{104}$.

Quasi-2D perovskite films consist of perovskite phases with multi-dimensions, which combines the advantages of both pure 3D and colloidal perovskites. Quasi-2D blue PeLEDs have achieved continuous breakthrough in optimizing device performance and stability. However, there is still much room for the further improvement, with research directions in the following aspects: 1) Optimizing the phase distribution. The category and molar ratio of the bulk cations play a key role in modifying the phases distribution. Bulk cations with phosphonate radical are the hotspot now, which should be thoroughly investigated. 2) Avoiding the Efficiency roll-off issue. 

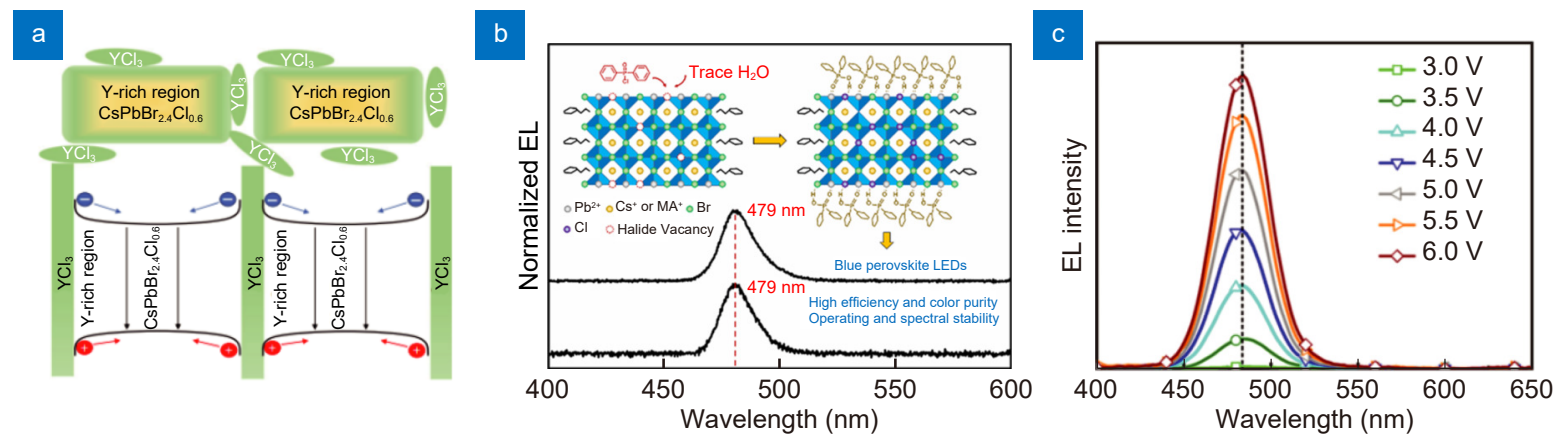

Fig. 7 | Spectral stability modification strategies for quasi-2D perovskite and their PeLEDs. (a) Schematic diagram of the yttrium gradient distribution in the $\mathrm{CsPbBr}_{3}: \mathrm{PEACl}(1: 1)$ film and its function in increasing the bandgap around the grain surface. (b) Stable EL spectra of DPPO$\mathrm{Cl}$-treated PeLEDs before and after operation. The inset is the schematic diagram of the mechanism of the DPPOCl induced chlorides-insertionimmobilization process. (c) EL spectra of moisture-treated blue emissive device operated under a different bias with moral ratio of $\mathrm{CsBr}_{\mathrm{B}} \mathrm{PbBr}_{2}$ of 2.2. Figure reproduced with permission from: (a) ref. ${ }^{98}$, Springer Nature; (b) ref. ${ }^{99}$, American Chemical Society; (c) ref. ${ }^{104}$, American Chemical Society.

The mechanism and strategies focus on this efficiency roll-off issue at high current density is rarely discussed in optimizing blue PeLEDs. The Auger recombination can be a possible mechanism for the efficiency roll-off issue at high current density, other reasonable explanations as well as optimization strategies should be provided to further improve the device performance of quasi-2D blue PeLEDs.

\section{Challenges and optimization strategies for device structures of blue PeLEDs}

The most commonly used device structure of PeLEDs is the "sandwich-like" structure with the CTLs inserted between the electrodes and the perovskite film. The HTL/ETL materials can prevent the leakage current caused by direct metal contact and provide additional energy levels to reduce the large energy offset between the electrode and the perovskite film, which facilitates charge injection. Compared with those of red and green PeLEDs, the device structure design of blue PeLEDs is more complicated. This is because the energy offset between the electrode and blue perovskites is much larger than those of its red and green counterparts. Such an energy offset severely hinders charge injection, especially hole injection, as the VBM of blue perovskite can be as deep as upto $7.1 \mathrm{eV}^{75}$, leaving an energy barrier of $2.4 \mathrm{eV}$ when the perovskite film is in direct contact with the ITO anode (work function (WF) of $4.7 \mathrm{eV}$ ). Moreover, the most commonly used HTL materials, such as PEDOT:PSS, can cause severe nonradiative recombination at the interface because of the intrinsic properties of exciton quenching and low WF $(\sim 5 \mathrm{eV})$. As the device structure has a significant effect on the device performance, strategies for better device structure design should be provided to fabricate high-efficiency blue PeLEDs. In this section, the optimization strategies for blue PeLEDs are summarized on the basis of three aspects: assembly of CTL materials, multiple HTLs, and interface modification.

\section{Single-layered electron/hole transport optimization strategy}

The single-layered electron/hole transport optimization strategy refers to the assembly of CTL materials with suitable energy levels and high charge carrier mobility to substitute for traditional organic counterparts, such as PEDOT:PSS and 2,2',2"-(1,3,5-benzinetriyl)-tris (1phenyl-1-H-benzimidazole) (TPBi), for the purpose of optimizing the charge transport/injection process and increasing the charge carrier concentration in perovskite films. Yao et al. applied $\mathrm{NiO}_{x}$ as the HTL material to fabricated blue PeLEDs for the first time, with an EQE of 0.07\% $\%^{51}$. Compared with PEDOT:PSS, $\mathrm{NiO}_{x}$ has features of wide bandgap and low highest occupied molecular orbital (HOMO) position, which not only promotes the hole transport but also confines the injected electrons inside the perovskite film. Xing et al. studied the effect of HOMO position of HTL materials on the turn-on voltage (defined as the voltage at luminance of $1 \mathrm{~cd} / \mathrm{m}^{2}$ ) of blue PeLEDs. The devices with PEDOT:PSS, $\mathrm{NiO}_{x}$, and PVK as the HTL materials showed gradually decreasing turn-on voltages of $6.2 \mathrm{~V}, 4.7 \mathrm{~V}$, and $3.8 \mathrm{~V}$, respectively. From these results we can conclude that the turn-on voltage of blue PeLEDs is mainly related to the 
energy offset between HTLs and the perovskite film ${ }^{91}$. Kim et al. adopted high electron mobility metal oxide $\mathrm{ZnO}$ and high hole mobility $\left(\mu_{\mathrm{h}} \approx 2.7 \times 10^{-3} \mathrm{~cm}^{2} \cdot \mathrm{V}^{-1} \cdot \mathrm{s}^{-1}\right)$, low HOMO $(\sim 5.4 \mathrm{eV})$ conductive polymer a-NPD as the ETM/HTM. The blue PeLED showed a decreased turnon voltage of $3.2 \mathrm{~V}$ with an $\mathrm{EQE}$ of $1.7 \%$, which originated from the promoted charge transport efficiency and balanced electron and hole current ${ }^{38}$. Yusoff et al. designed an all-inorganic inverted device structure by applying AZO:Cs and CuS-GaSnO as the ETL and HTL materials, respectively ${ }^{105}$. The electron/hole mobility of AZO:Cs/CuS-GaSnO is higher than that of traditional organic transport materials, such as PEDOT:PSS and TPBi. The conductivity of AZO:Cs and CuS-GaSnO can be further improved by increasing the ratio of $\mathrm{Cs}$ to $\mathrm{Ga}$. Also, the energy level alignment is optimized with AZO:Cs and CuS-GaSnO as the ETL and HTL materials, respectively, further increasing the charge injection rate. The operating lifetime is also improved as these inorganic CTL materials exhibited better water stability. The encapsulated PeLEDs can operate continuously for $16 \mathrm{~h}$ at an initial luminance of $1000 \mathrm{~cd} / \mathrm{m}^{2}$. Yang et al. adopted novel organic ETM 2,4,6-Tris(3-(diphenylphosphoryl) phenyl)-1,3,5-triazine (PO-T2T) with a high electron mobility of $1.1 \times 10^{-4} \mathrm{~cm}^{2} \cdot \mathrm{V}^{-1} \cdot \mathrm{s}^{-1}$ to replace the traditional ETM TPBi with a low electron mobility of $1 \times 10^{-6}$ $\mathrm{cm}^{2} \cdot \mathrm{V}^{-1} \cdot \mathrm{s}^{-1}$. By this substitution, the as-prepared blue $(477 \mathrm{~nm})$ PeLED showed an improved EQE from 1.19\% to $1.96 \%{ }^{45}$.

\section{Multi-layered hole transport optimization strategy}

The multiple HTL strategy refers to inserting two or more layers of hole transport materials between the anode and perovskite film. Hoye et al. found that there exists large energy offset $(\sim 1.3 \mathrm{eV})$ between PEDOT:PSS and colloidal PNLs, which not only hindered hole injection rate but also triggered exciton dissociation at the PEDOT:PSS/EML interface. By inserting layers of poly(triarylamine) polymers like TFB or poly $\left(\mathrm{N}, \mathrm{N}^{\prime}-\right.$ bis(4-butylphenyl)-N,N'-bisphenylbenzidine) (POLYTPD), the energy barrier as well as the nonradiative recombination rate at the interface were minimized. The EQE of as-prepared deep-blue (464 $\mathrm{nm}$ ) and sky-blue (489 nm) PeLEDs improved two orders of magnitude from $0.007 \%$ to $0.3 \%$ and $0.004 \%$ to $2.4 \%$, respectively ${ }^{106}$. Besides the low HOMO and high exciton quenching rates, the intrinsic ionic property of PEDOT:PSS can speed up the crystallization rate of $3 \mathrm{D}$ phases in quasi-
2D perovskite films and lead to red-shifting of spectra. To solve this problem, Ma et al. inserted a thin layer of POLY-TPD on top of the PEDOT:PSS by spinning-cast method, which prevented the red-shift of EL spectra from $479 \mathrm{~nm}$ to $489 \mathrm{~nm}$ when applying PEDOT:PSS/PFI as the HTL materials. Anisole was selected as the solvent to dissolve the POLY-TPD, with trace amounts of anisole residue remaining in POLY-TPD films that can be beneficial for the film morphology of perovskites ${ }^{99}$. The bilayer structure of PEDOT:PSS/poly(9-vinlycarbazole) (PVK) is the most commonly used device structure in the fabrication of colloidal PQD- and PNL-based PeLEDs. PVK has a suitable energy level with deep HOMO position $(\sim 5.8 \mathrm{eV})$ that not only reduces the hole injection barrier but also blocks electrons inside the perovskite film. Deng et al. blended conductive molecular TAPC $(w t \%=10 \%)$ into PVK to further increase the hole current density of PeLEDs ${ }^{95}$.

An HTM tri-layer strategy is widely applied in the fabrication of deep-blue PeLEDs with emission peak wavelength less than $470 \mathrm{~nm}$. Kumar et al. fabricated trilayer HTMs based on the PEDOT:PSS/PVK bilayer structure. Donor materials like 4,4'-bis(N-carbazolyl)1,1'-biphenyl (CBP) were inserted on top of the PVK layer, and the excitons could transfer and recombine efficiently in the perovskite film through the Förster resonance energy transfer process (FRET). They believed that a large spectral overlap between the absorption spectra of the acceptor materials (perovskites) and the donor emission spectra is a prerequisite for the FRET process, which gives guidance for selecting suitable HTL materials ${ }^{74}$. Gangishetty et al. substituted $\mathrm{NiO}_{x}$ with PEDOT:PSS/ TFB/PFI tri-layer HTMs, which not only enhanced the hole transport rate but also prevented nonradiative recombination at the interface due to wide existence of defect sites at the surface of $\mathrm{NiO}_{x}$. Besides, the PFI layer exhibited a low surface energy, which not only improved the film quality of PQD films but also weakened the emission from the TFB layers. The as-prepared $\mathrm{CsPbBr}{ }_{x} \mathrm{Cl}_{3-x}$-QD-based PeLEDs showed an improved EQE from $0.07 \%$ when using $\mathrm{NiO}_{x}$ as the $\mathrm{HTL}$ to $0.5 \%$, reaching a maximum luminance of $111 \mathrm{~cd} / \mathrm{m}^{2}$ at a peak wavelength of $469 \mathrm{~nm}^{107}$. Hou et al. adopted the same device structure of PEDOT:PSS/TFB/PFI to improve the hole injection rate of Mn-doped PQD PeLEDs with the emission peak in deep-blue region $(466 \mathrm{~nm})$. However, there were still small amounts of excitons radiatively recombined at the TFB layer, which degraded the color 
purity of devices ${ }^{64}$. To eliminate the EL from the TFB layer, Shynkarenko et al. fabricated a tri-layer HTM structure of PEDOT:PSS/POLY-TPD/PVK. Deep-blue PeLEDs (463 nm) with narrow EL spectra were fabricated, which originated from the deeper VBM position of PVK $(\sim 5.8 \mathrm{eV})$ compared with that of TFB $(\sim 5.4 \mathrm{eV})$, which can confine the excitons to the perovskite film ${ }^{54}$. Other deep-blue PeLEDs (463 nm) with high EL color purity were fabricated by Ochsenbein et al. They adopted tri-layer HTMs of PEDOT:PSS/POLY-TPD/CBP to improve the hole injection. The obtained devices showed a low turn-on voltage of only $2.9 \mathrm{~V}$, which is slightly higher than the bandgap of deep-blue $\mathrm{CsPb}(\mathrm{Br} / \mathrm{Cl})_{3}$ QDs $(2.68 \mathrm{eV})$ as the perovskite film. The FWHM of the EL spectra was only $14 \mathrm{~nm}$, indicating high-purity emission $^{49}$.

\section{Interface modification strategy}

The interface modification strategy refers to inserting a functional layer between the CTL and emissive layer to improve the device performance. Those functional materials cannot be a CTL solely; however, the charge transport efficiency, perovskite film quality, and nonradiative recombination at the interface can be optimized by the modification of functional materials. Cheng et al. modified $\mathrm{ZnO}$ with polyethylenimine ethoxylated (PEIE) to decrease the work function of $\mathrm{ZnO}$. The energy offset between the $\mathrm{ZnO}$ and perovskite film was reduced, which is beneficial for electron injection. Sky-blue (490 nm) PeLEDs based on PEIE-modified ZnO substrates showed a low turn-on voltage of $2.9 \mathrm{~V}^{82}$. Chiba et al. inserted a layer of oleylammonium chloride (OAM$\mathrm{Cl}$ ) between the TFB and PQDs emissive layer, with the EQE of device increased from $0.49 \%$ to $1.1 \%$. Further measurement confirmed that the OAM-Cl interfacial layer does not influence the optical property of $\mathrm{PQD}$ emissive films above, and the improved efficiency was mainly attributed to the balanced charge injection ${ }^{69}$. Wang et al. modified $\mathrm{NiO}_{x}$ with a thin layer of $\mathrm{LiF}$, the device structure is shown in Fig. 8(a). The LiF-modified $\mathrm{NiO}_{x}$ showed better hydrophilicity, which ameliorated the morphology of quasi-2D perovskite films. The PLQY and steady-state PL intensity increased with the modification of LiF, indicating that the trap-mediated non-radiative recombination at the surface of $\mathrm{NiO}_{x}$ is suppressed efficiently. Moreover, balanced charge injection can be achieved by increasing the thickness of LiF from $0.5 \mathrm{~nm}$ to $1.5 \mathrm{~nm}$, as the fabricated quasi-2D PeLEDs were proved to be hole-dominated devices, and the hole current density decreased as the thickness of LiF increased. The as-prepared sky-blue PeLEDs showed a maximum luminance of $1440 \mathrm{~cd} / \mathrm{m}^{2}$ with an EQE of $0.52 \%{ }^{86}$. A similar strategy was proposed by Ren et al. by adopting a thin polymer layer of PSSNa to modify the $\mathrm{NiO}_{x}$ surface. With the modification of PSSNa, the nonradiative recombination in $\mathrm{NiO}_{x}$ was fully suppressed, with the PLQY of perovskite films improved from $9.8 \%$ to $12.7 \%$. Simultaneously, dense and uniform quasi-2D perovskite films were formed due to the increased hydrophilicity of $\mathrm{NiO}_{x}$. Moreover, the dipole feature of PSSNa lowered the $\mathrm{VBM}$ of $\mathrm{NiO}_{x}$ from $5.51 \mathrm{eV}$ to $5.85 \mathrm{eV}$, which reduced the energy offset between $\mathrm{NiO}_{x}$ and the perovskite film and facilitated hole injection. By further optimizing the thickness of PSSNa ( 2 nm), sky-blue PeLEDs showed a maximum luminance of $5737 \mathrm{~cd} / \mathrm{m}^{2}$ with a turn-on voltage of $3.31 \mathrm{~V}^{108}$. The interface dipole effect was also reported by Shin et al. They inserted a halide functionalized layer, poly[(9,9-bis(3'-(N,N-dimethylamino)propyl)2,7-fluorene)-alt-2,7-(9,9-dioctylfluorene)] (PFN-X, X = $\mathrm{I}, \mathrm{Br}, \mathrm{Cl}$ ), to modify the underlying POLY-TPD layer. By adopting PFN-X interlayer with different counter anions, the HOMO of the POLY-TPD can be adjusted from 5.45 to $5.80 \mathrm{eV}$. Besides, the electron blocking property of PFN-X was confirmed to be increased with the PLQY of the PQD films increased from $0.94 \%$ to $2.01 \%$. The optimized PeLEDs displayed a maximum EQE of $1.34 \%$, with a stable EL peak position at $470 \mathrm{~nm}^{109}$. Wang et al. modified PEDOT:PSS with a thin layer of $\mathrm{RbCl}$. The device structure and the cross-section picture of PeLED are shown in Fig. 8(c) and Fig. 8(d), respectively. The steady-state PL intensity of pure 3D perovskite films received a 48-fold promotion when the substrate changed from PEDOT:PSS to PEDOT:PSS/RbCl, indicating that the exciton quenching at the interface was hindered effectively by the modification of RbCl. Sky-blue (484 nm) pure 3D PeLEDs based on this $\mathrm{RbCl}$ modification strategy showed a maximum luminance of $9243 \mathrm{~cd} / \mathrm{m}^{2}$ with a low turn-on voltage of only $2.6 \mathrm{~V}$, which is the highest luminance blue PeLED based on pure 3D perovskite film. $\mathrm{CsBr}$ and $\mathrm{RbBr}$ can also act as interface modification layers to improve the EL performance, which implies that the modification of inorganic halides may be a universal method for optimizing blue PeLEDs ${ }^{110}$. Yuan et al. fabricated sky-blue $(484 \mathrm{~nm})$ PeLEDs based on an insulator-perovskite-insulator device structure without applying additional HTL 
a
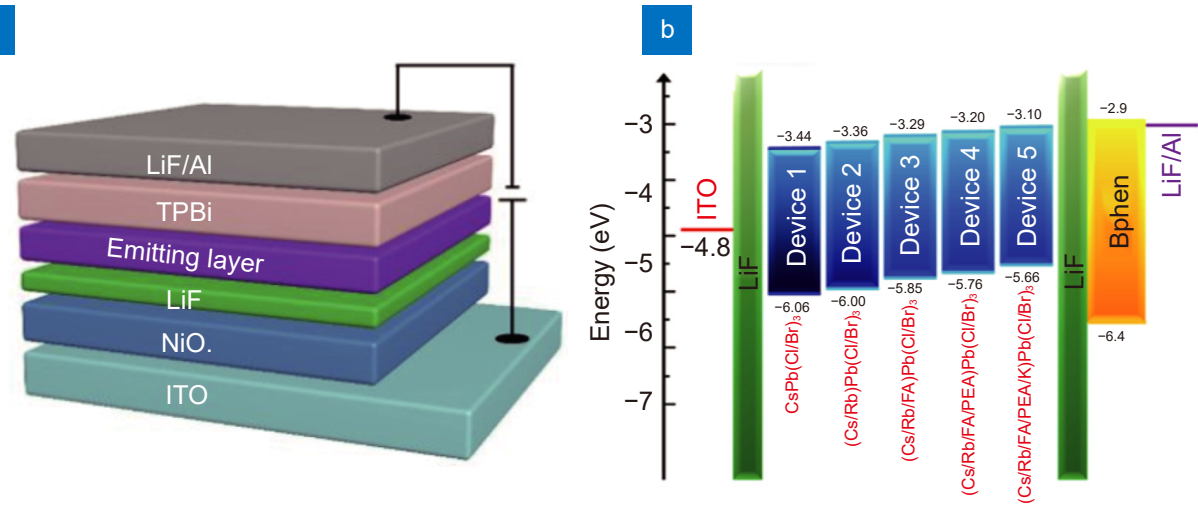

c

d

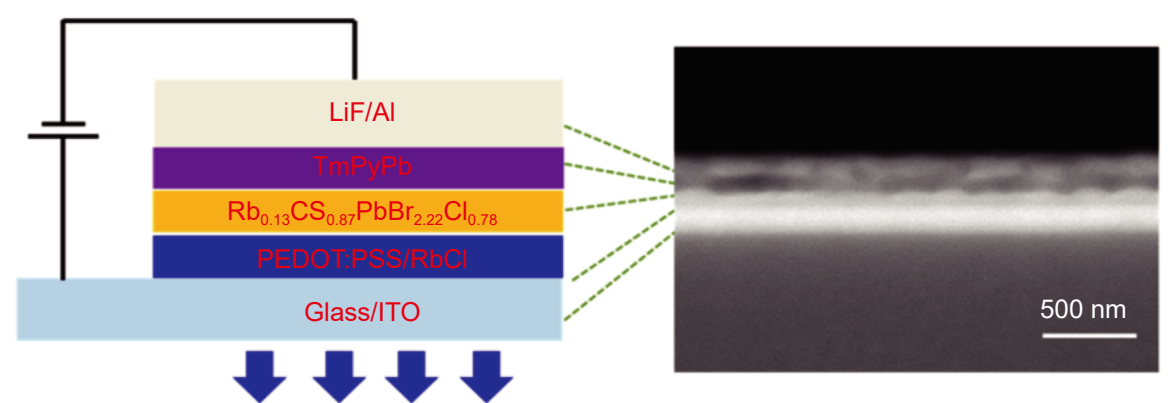

Fig. 8 | Interface modification strategies for blue PeLEDs. (a) Device structure of quasi-2D blue PeLEDs with LiF as the interface modification layer. (b) Energy level alignment diagram of blue PeLEDs with device structure of LiF-perovskite-LiF. (c) Device structure and (d) cross-section picture of quasi-2D PeLEDs with $\mathrm{RbCl}$ as the interface modification layer. Figure reproduced from: (a) ref. ${ }^{86}$, American Chemical Society; (b) ref. ${ }^{40}$, American Chemical Society; (c) and (d) ref. ${ }^{110}$, American Chemical Society.
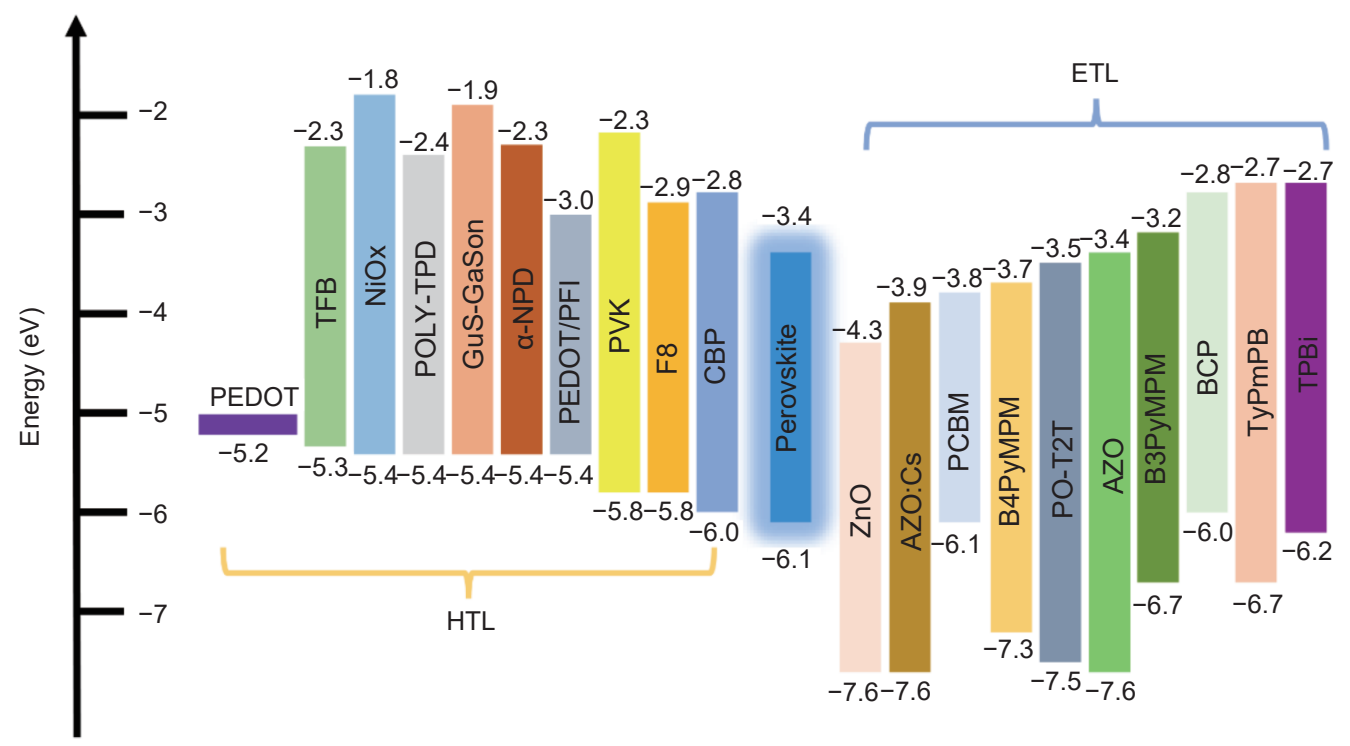

Fig. 9 | Energy level alignments of various ETL, HTL, and emissive layer materials.

materials. A thin layer of $\mathrm{LiF}$ was inserted on both sides of the emissive layers to form a quantum well structure that can confine the excitons inside the emissive layer and prevent exciton quenching at the interface, as shown in the energy band alignment in Fig. 8(b). The optimized devices showed a maximum luminance is 4015 $\mathrm{cd} / \mathrm{m}^{2}$ with improved operating lifetime of $300 \mathrm{~min}$ at a constant current of $25 \mathrm{~mA} / \mathrm{cm}^{2}{ }^{40}$.

An energy diagram of the commonly used ETL/HTL materials is shown in Fig. 9. In short, the key factors for device structure design of blue and deep-blue PeLEDs are as follows: 1) selecting carrier transport materials with matching energy levels against the blue and deep-blue perovskites, 2) increasing the carrier mobility 
of HTL/ETL materials to facilitate carrier transport, 3) improving the intrinsic stability of HTL/ETL materials, and 4) modifying the interface of CTLs and the perovskite film.

\section{Conclusion}

In this paper, the recent achievements of blue PeLEDs were reviewed from two aspects: optimization strategy for blue-emitting perovskite films as well as the design of device structure. For achieving high-quality perovskite films with low defect density, pure emission, and good film morphology, different optimization strategies focusing on either the perovskite precursor or perovskite film formation process have been proposed based on the features of the four kinds of perovskite materials. For achieving blue-emitting PeLEDs with high charge carrier concentration in perovskite films, balanced charge injection rate, and low interface energy loss, the device structure should be well-designed. Here, we present a discussion on the features and future development directions of the four kinds of perovskite materials as well as the design of device structure.

\section{Features and outlook for pure 3D perovskites}

Pure 3D perovskites show optimal conductivity, which is beneficial for charge carrier injection into the perovskite film. However, the lowest $E_{\mathrm{B}}$ as well as the significant number of defect sites degrade the PLQY of pure 3D perovskite films, which limits the efficiency of PeLEDs based on pure $3 \mathrm{D}$ perovskites. Besides, the fabrication of pure 3D perovskite-based PeLEDs in the of deep-blue spectral region $(<470 \mathrm{~nm})$ remains a challenge. Due to the poor solubility of $\mathrm{PbCl}_{2}$, most blue-emitting $3 \mathrm{D}$ perovskites have mixed halide components, which easily succumb to phase segregation during device operation. The most effective way to improve the stability of pure 3D PeLEDs is mixing A site cations, which should be regarded as a potential development direction for stable pure 3D PeLEDs in deep-blue region.

\section{Features and outlook for colloidal PQDs and PNLs}

Compared with pure 3D PeLEDs, colloidal PQDs show better water and oxygen stability due to the existence of long-chain alkyl ligands at the surface of PQDs. These ligands can not only protect the PQDs from water and oxygen but also passivate the defect sites at the surface, especially halogen vacancies. PLQYs of colloidal PQDs have reached almost $100 \%$. By precisely controlling the reactive conditions and concentrations of various precursors, colloidal PNLs can be synthesized with precise thickness. The emission spectra can be tuned from 405 $\mathrm{nm}$ to $490 \mathrm{~nm}$ by adjusting the layer thickness of colloidal PNLs from $n=1$ to 5 due to the quantum confinement effect. However, these two kinds of colloidally synthesized perovskites have similar inherent shortcomings. First, the PLQYs of colloidal PQDs or PNLs in films are much lower than that in dispersion. The main reason for this degradation is the severe ligand loss during the purification and film formation processes, especially in PNLs with larger surface/volume ratios. Also, the insulating property of the alkyl chain hinders the charge injection into these colloidal perovskite materials. To improve the PLQY in film state, the ligand loss problem should be solved. Ligands with better affinity should be selected, and other modification strategies, such as post-treatment and in-situ passivation should be applied to enhance the electronic interaction between ligands and perovskites. To improve the conductivity of colloidal perovskite films, ligands with shorter chain lengths should be adopted. The most promising way to promote the conductivity of colloidal perovskite films is to use metal ions as capping ligands. As $\mathrm{Na}^{+}, \mathrm{K}^{+}$and $\mathrm{Ca}^{2+}$ are metal ions that has been applied to modify blue PQDs as capping ligands to fabricate PeLEDs, other metals ions should be exploited.

\section{Features and outlook for Quasi-2D perovskites}

Quasi-2D perovskites may be the most promising materials to fabricate high-efficiency blue PeLEDs. By using bulk organic ammonium cations as the ligands, quasi-2D films composed of multi-dimensional phases of perovskite can be achieved in solid film state. The existence of multi-dimensional phases enables quasi-2D perovskites to be a bridge that connects pure $3 \mathrm{D}$ perovskites and colloidal 2D PNLs. High conductivity as well as high $E_{\mathrm{B}}$ properties can be obtained simultaneously, which is the most promising feature of quasi-2D perovskite films. For the future of quasi-2D blue PeLEDs, the dimension distribution of perovskite films should be optimized to diminish the energy loss during the energy transfer process. Strategies like mixing A site cations, mixing bulk cations, and additives engineering, can be utilized to further promote the efficiency and stability of devices based on quasi-2D perovskites. Some novel film formation 
Opto-Electronic Advances https://doi.org/10.29026/oea.2021.200019

Table 1 | Summary of the main blue PeLEDs.

\begin{tabular}{|c|c|c|c|c|c|c|c|c|}
\hline \multirow[b]{2}{*}{ Year } & \multirow[b]{2}{*}{ Type } & \multirow[b]{2}{*}{ Device Structure } & \multicolumn{6}{|c|}{ Device performance } \\
\hline & & & $\begin{array}{l}\lambda_{(\mathrm{EL})} / \mathrm{FWHM} \\
(\mathrm{nm} / \mathrm{nm})\end{array}$ & EQE (\%) & $\begin{array}{l}\text { Max lumin } \\
\left(\mathrm{cd} / \mathrm{m}^{2}\right)\end{array}$ & $\mathrm{CE}(\mathrm{cd} / \mathrm{A})$ & $V_{\mathrm{T}}(\mathrm{V})$ & Ref \\
\hline 2016 & 3D Bulk & ITO/PEDOT:PSS/Pero/TPBi/Ca/AI & $490 / 30$ & - & 154 & 0.08 & 4.1 & Ref. ${ }^{37}$ \\
\hline 2017 & 3D Bulk & ITO/ZnO/Pero/NPD/MoO3/AI & $475 /-$ & 1.7 & 3567 & 11.31 & 3.2 & Ref. ${ }^{38}$ \\
\hline 2018 & 3D Bulk & ITO/AZO:Cs/Pero/CuS-GaSnO/WO3/Ag & $475 / 19$ & 2.58 & 6426 & 15.21 & 3.1 & Ref. ${ }^{105}$ \\
\hline 2019 & 3D Bulk & ITO/PEDOT:PSS/Pero/TPBi/LiF/AI & $490 /$ & 0.58 & 1470 & - & $\sim 4.2$ & Ref. ${ }^{41}$ \\
\hline 2019 & 3D Bulk & ITO/PEDOT:PSS/Pero/TyPmPb/LiF/AI & $468 / 20,492 / 20$ & $0.062,0.17$ & 112,244 & $0.06,0.32$ & $4.4,3.4$ & Ref. ${ }^{39}$ \\
\hline 2019 & 3D Bulk & ITO/NiOx/Pero/TPBi/LiF/Al & $\begin{array}{c}456 / 24,468 / 23 \\
480 / 20\end{array}$ & $0.15,0.38,0.05$ & $51,121,87$ & $\begin{array}{l}0.07,0.21 \\
0.04\end{array}$ & $4,3.5,4$ & Ref. $^{42}$ \\
\hline 2020 & 3D Bulk & $\begin{array}{c}\text { ITO/PEDOT:PSS/RbCl/pero/Tmpypb/Li } \\
\text { F/Al }\end{array}$ & $484 / 12$ & 1.66 & 9243 & 2.15 & 2.6 & Ref. ${ }^{110}$ \\
\hline 2020 & 3D Bulk & ITO/LiF/pero//LiF/Bphen/Al & $484 / 22$ & 2.01 & 4015 & 2.11 & $\sim 4$ & Ref. ${ }^{40}$ \\
\hline 2015 & NC & ITO/PEDOT:PSS/PVK/Pero/TPBi/LiF/AI & $455 / 20$ & 0.07 & 742 & 0.14 & 5.1 & Ref..$^{43}$ \\
\hline 2016 & NC & ITO/ZnO/Pero/TFB/MoO3/Ag & $480 / 17$ & 0.0074 & 8.7 & - & 5.5 & Ref..$^{48}$ \\
\hline 2016 & NC & ITO/PEDOT:PSS/PVK/Pero/TPBi/LiF/AI & $445 / 30$ & 1.18 & 2473 & - & $\sim 7.8$ & Ref..$^{50}$ \\
\hline 2016 & NC & ITO/PEDOT:PSS/PVK/Pero/TPBi/LiF/AI & $490 / 19$ & 1.9 & 35 & - & 3 & Ref..$^{45}$ \\
\hline 2017 & NC & ITO/NiOx/Pero/TPBi/LiF/Al & $470 / 20$ & 0.07 & 350 & 0.18 & $\sim 5$ & Ref. ${ }^{51}$ \\
\hline 2018 & NC & ITO/PEDOT:PSS/PVK/Pero/TPBi/LiF/AI & $465 / 15.6$ & $\sim 0.2$ & $\sim 100$ & 0.1 & $\sim 6$ & Ref..$^{52}$ \\
\hline 2018 & NC & ITO/TFB/PFI/Pero/TPBi/LiF/AI & $469 / 25$ & 0.5 & 111 & - & $\sim 5$ & Ref. ${ }^{107}$ \\
\hline 2018 & NC & ITO/POLY-TPD/Pero/TPBi/LiF/AI & $466 / 23,502 / 23$ & $0.61,3.6$ & 39,750 & $8.5 @ 502$ nm & $\sim 4$ & Ref. 63 \\
\hline 2018 & NC & ITO/PEDOT/TFB/PFI/Pero/TPBi/LiF/AI & $466 / 17.1$ & 2.12 & 389 & - & 4 & Ref. ${ }^{64}$ \\
\hline 2019 & NC & $\begin{array}{c}\text { ITO/PEDOT:PSS/POLY- } \\
\text { TPD/CBP/Pero/B3PyMpM/LiF/Al }\end{array}$ & $463 / 14$ & 1.4 & 318 & - & 2.9 & Ref. ${ }^{49}$ \\
\hline 2019 & NC & $\begin{array}{l}\text { ITO/PEDOT:PSS/POLY- } \\
\text { TPD/Pero/TPBi/LiF/AI }\end{array}$ & $\begin{array}{c}458 / 18,469 / 18 \\
479 / 18\end{array}$ & $0.1,0.44,0.86$ & $\begin{array}{l}3.87,11.95 \\
29.95\end{array}$ & $\begin{array}{l}0.06,0.28 \\
\quad 0.77\end{array}$ & $4.5,4,3.5$ & Ref..$^{53}$ \\
\hline 2019 & NC & $\begin{array}{c}\text { ITO/PEDOT:PSS/POLY- } \\
\text { TPD/PVK/Pero/B3PYMPM/TPBi/LiF/AI }\end{array}$ & $\begin{array}{c}463 / 18,476 / 18 \\
490 / 18\end{array}$ & $1.03,2.25,3.5$ & 193, 678, 2063 & - & 3-3.4 & Ref. ${ }^{54}$ \\
\hline 2019 & NC & $\begin{array}{l}\text { ITO/PEDOT:PSS/POLY- } \\
\text { TPD/Pero/TPBi/LiF/AI }\end{array}$ & $476 / 20$ & 1.96 & 212.9 & - & 3.2 & Ref. ${ }^{47}$ \\
\hline 2020 & NC & ITO/TFB/PFI/Pero/3TPYMP/Liq/AI & $471 / 17$ & 6.2 & 465 & - & - & Ref. ${ }^{68}$ \\
\hline 2020 & NC & $\begin{array}{c}\text { ITO/PEDOT:PSS/TFB/PFI/Pero/TPBi/Li } \\
\text { F/Al }\end{array}$ & $470 /-$ & 2.4 & 612 & - & 3.2 & Ref. ${ }^{65}$ \\
\hline 2020 & NC & $\begin{array}{l}\text { ITO/PEDOT:PSS/POLY- } \\
\text { TPD/Pero/TPBi/Ca/Ag }\end{array}$ & $\begin{array}{l}469 / 18,479 / 18 \\
489 / 18,496 / 18\end{array}$ & $0.65,1,1.8,2.6$ & $30,119,182,603$ & $\begin{array}{c}0.47,0.94 \\
2.4,4.5\end{array}$ & $\begin{array}{l}3.8,3.2 \\
3.4,3.2\end{array}$ & Ref..$^{55}$ \\
\hline 2020 & NC & ITO/NiOx/Pero/TPBi/LiF/Al & $445 /-$ & 1.12 & 262.6 & & 4.5 & Ref. $^{70}$ \\
\hline 2020 & NC & $\begin{array}{l}\text { ITO/PEDOT:PSS/POLY- } \\
\text { TPD/Pero/TPBi/LiF/AI }\end{array}$ & $459 /-$ & 0.3 & 76 & 0.3 & - & Ref. ${ }^{66}$ \\
\hline 2020 & NC & $\begin{array}{c}\text { ITO/PEDOT:PSS/PTAA/Pero/TPBi/LiF/ } \\
\mathrm{Al}\end{array}$ & $479 / 20$ & 12.3 & $\sim 600$ & - & 2.8 & Ref..$^{48}$ \\
\hline 2020 & NC & $\begin{array}{l}\text { ITO/PEDOT:PSS/POLY- } \\
\text { TPD/PVKPero/TyPmPB/LiF/AI }\end{array}$ & $\begin{array}{l}462 / 19,465 / 19 \\
468 / 20,470 / 21\end{array}$ & $\begin{array}{l}0.77,0.92,1.53 \\
2.15\end{array}$ & $450,518,620,507$ & $\begin{array}{c}4.5,5.1 \\
5.4,6.0\end{array}$ & $\begin{array}{l}4.4,4.6 \\
4.8,4.9\end{array}$ & Ref. ${ }^{67}$ \\
\hline 2020 & NC & $\begin{array}{l}\text { ITO/PEDOT:PSS/TFB/OAM- } \\
\text { CIPero/TPBi/Liq/Al }\end{array}$ & $456 / 16$ & 1.1 & 43.2 & - & 5.4 & Ref. ${ }^{69}$ \\
\hline 2020 & NC & $\begin{array}{c}\text { ITO/PEDOT:PSS/TFB/PFI/Pero/TPBi/Li } \\
\text { F/Al }\end{array}$ & $463 / 17$ & 3.3 & 569 & - & 4 & Ref. ${ }^{46}$ \\
\hline 2020 & NC & $\begin{array}{c}\text { ITO/PEDOT:PSS/POLY-TPD/PFN- } \\
\text { X/Pero/TPBi/LiF/Al }\end{array}$ & $470 / 17$ & 1.34 & 46.7 & 1.24 & 3 & Ref. ${ }^{109}$ \\
\hline 2016 & NPL & $\begin{array}{l}\text { ITO/PEDOT:PSS/PVK/Host } \\
\text { layer/Pero/TPBi/LiF/Al }\end{array}$ & $\begin{array}{c}432 / 25,456 / 25 \\
492 / 25\end{array}$ & $0.23,0.0240 .004$ & 8.5 & - & 3.5 & Ref. ${ }^{74}$ \\
\hline 2018 & NPL & $\begin{array}{l}\text { ITO/PEDOT:PSS/POLY- } \\
\text { TPD/Pero/TPBi/LiF/AI }\end{array}$ & $480 /-$ & $\sim 0.1$ & 25 & - & 4.6 & Ref. ${ }^{77}$ \\
\hline 2018 & NPL & $\begin{array}{l}\text { ITO/PEDOT:PSS/POLY- } \\
\text { TPD/Pero/TPBi/Ca/Ag }\end{array}$ & $464 / 20$ & 0.057 & 38 & - & 3.6 & Ref. $^{76}$ \\
\hline
\end{tabular}

200019-24 
Opto-Electronic Advances https://doi.org/10.29026/oea.2021.200019

Table 1 (Continued)

\begin{tabular}{|c|c|c|c|c|c|c|c|c|}
\hline \multirow[b]{2}{*}{ Year } & \multirow[b]{2}{*}{ Type } & \multirow[b]{2}{*}{ Device Structure } & \multicolumn{6}{|c|}{ Device performance } \\
\hline & & & $\begin{array}{c}\lambda_{(\mathrm{EL} / \mathrm{FWHM}} \\
(\mathrm{nm} / \mathrm{nm})\end{array}$ & EQE (\%) & $\begin{array}{l}\text { Max lumin } \\
\left(\mathrm{cd} / \mathrm{m}^{2}\right)\end{array}$ & $C E(c d / A)$ & $V_{T}(\mathrm{~V})$ & Ref \\
\hline 2018 & NPL & $\begin{array}{l}\text { ITO/PEDOT:PSS/POLY- } \\
\text { TPD/Pero/TPBi/LiF/AI }\end{array}$ & $463 / 12$ & 0.124 & 62 & 0.117 & 4.2 & Ref. $^{78}$ \\
\hline 2019 & NPL & $\begin{array}{l}\text { ITO/PEDOT:PSS/TFB or POLY- } \\
\text { TPD/Pero/TPBi/Ca/Ag }\end{array}$ & $464 / 16,489 / 25$ & $0.3,0.55$ & 48,40 & $0.3,1.1$ & 4 & Ref. ${ }^{106}$ \\
\hline 2019 & NPL & $\begin{array}{l}\text { ITO/PEDOT:PSS/POLY- } \\
\text { TPD/Pero/TPBi/LiF/AI }\end{array}$ & $464 / 18,490 / 18$ & $0.11,0.87$ & 71,186 & - & 4 & Ref. $^{56}$ \\
\hline 2019 & NPL & $\begin{array}{c}\text { ITO/PEDOT/POLY- } \\
\text { TPD/CBP/Pero/TPBi/LiF/AI }\end{array}$ & $469 /-$ & 1.42 & 41.8 & - & 3 & Ref. $^{75}$ \\
\hline 2020 & NPL & $\begin{array}{c}\text { ITO/POLY- } \\
\text { TPD/MoO3/Pero/TyPmPB/LiF/Al }\end{array}$ & $439 / 14$ & 0.14 & 9.7 & - & 3.6 & Ref. $^{83}$ \\
\hline 2016 & Quasi-2D & ITO/PEDOT:PSS/Pero/TPBi/Ca/AI & $410 / 14$ & 0.038 & - & - & 2.5 & Ref. ${ }^{94}$ \\
\hline 2016 & Quasi-2D & ITO/ZnO/PEIE/Pero/TFB/MoOx/AI & 491/broad & 0.015 & 186 & - & 2.9 & Ref. ${ }^{82}$ \\
\hline 2016 & Quasi-2D & $\begin{array}{c}\text { ITO/PEDOT:PSS/POLY-TPD/QW- } \\
\text { Pero/TPBi/LiF/AI }\end{array}$ & $468 / 28$ & 0.01 & 21 & 0.006 & 5.2 & Ref. $^{74}$ \\
\hline 2016 & Quasi-2D & ITO/PEDOT:PSS/Pero/TPBi/Ba/AI & $\begin{array}{l}462 / \text { broad, } \\
480 / \text { broad }\end{array}$ & $0.06,1.1$ & $1.62,19.25$ & $0.07,2.1$ & - & Ref. $^{79}$ \\
\hline 2017 & Quasi-2D & ITO/PEDOT:PSS/Pero/TypmpB/CsF/AI & $485 /$ broad & 2.6 & 200 & - & 3.4 & Ref. $^{84}$ \\
\hline 2018 & Quasi-2D & $\begin{array}{c}\text { ITO/PEDOT:PSS/NiOx/PVK/Pero/TPBi/ } \\
\text { LiF/Al }\end{array}$ & $490 / 28$ & 1.5 & 2480 & 2.8 & 5 & Ref. $^{91}$ \\
\hline 2018 & Quasi-2D & ITO/PEDOT:PSS/PVK/Pero/TPBi/LiF/AI & $465 / 23,487 / 22$ & $2.4,6.2$ & 962,3340 & 1 & $5,4.5$ & Ref. ${ }^{85}$ \\
\hline 2018 & Quasi-2D & ITO/NiOx/LiF/Pero/TPBi/LiF/Al & $\begin{array}{c}473 / 24 \\
481 / 24,490 / 24\end{array}$ & $0.16,0.25,0.52$ & $217,509,1446$ & - & 3.5 & Ref. $^{86}$ \\
\hline 2019 & Quasi-2D & ITO/PEDOT:PSS/Pero/TPBi/LiF/AI & $480 / 21$ & 5.7 & 3780 & 6.2 & 3.2 & Ref. $^{87}$ \\
\hline 2020 & Quasi-2D & $\begin{array}{c}\text { ITO/PVK:F4- } \\
\text { TCNQ/Pero/TPPO/TPBi/LiF/AI }\end{array}$ & $492 / 18$ & 8.2 & 1687 & 13.1 & $\sim 3.8$ & Ref. $^{88}$ \\
\hline 2019 & Quasi-2D & ITO/PEDOT:PSS/Pero/Tmpypb/LiF/AI & $475 / 25$ & 1.35 & 100.6 & $\sim 1$ & $\sim 3$ & Ref. ${ }^{89}$ \\
\hline 2019 & Quasi-2D & ITO/PVK/Pero/TPBi/LiF/Al & $474 / 34,484 / 34$ & $0.002,0.13$ & 4,45 & $0.002,0.14$ & $\sim 3$ & Ref. 102 \\
\hline 2019 & Quasi-2D & $\begin{array}{c}\text { ITO/PEDOT/PVK:10\%TAPC/Pero/TPBi/ } \\
\mathrm{Ca} / \mathrm{Ag}\end{array}$ & $410 / 11.6$ & 0.31 & 147.6 & 0.19 & 4.2 & Ref. ${ }^{95}$ \\
\hline 2019 & Quasi-2D & ITO/NiOx/PSSNa/Pero/TPBi/LiF/AI & $493 / 25$ & 1.45 & 5737 & 2.25 & 3.3 & Ref. ${ }^{108}$ \\
\hline 2019 & Quasi-2D & ITO/NiOx/TFB/PVK/Pero/TPBi/LiF/AI & $483 / 26$ & 9.5 & 700 & - & 3.3 & Ref. $^{90}$ \\
\hline 2019 & Quasi-2D & ITO/PVK/PFI/Pero/3TPYMB/Liq/Al & $465 / 21$ & 2.6 & 211 & $\sim 1.3$ & 3 & Ref. $^{92}$ \\
\hline 2019 & Quasi-2D & ITO/PEDOT:PSS/Pero/TPBi/LiF/AI & $468 / 25$ & 0.71 & 122 & 0.78 & 4.5 & Ref. $^{97}$ \\
\hline 2019 & Quasi-2D & $\begin{array}{l}\text { ITO/PEDOT:PSS or } \\
\text { NiOx/Pero/TPBi/LiF/Al }\end{array}$ & 480/-, 490/-, 499/- & $1.43,2.4,1.58$ & $919,2825,7759$ & $1.53,3.67$ & $\begin{array}{c}3.5,3.3 \\
4.4\end{array}$ & Ref. ${ }^{102}$ \\
\hline 2019 & Quasi-2D & ITO/PVK/Pero/PO-T2T/Liq/AI & $485 / 23$ & 2.62 & 1200 & - & 2.6 & Ref. ${ }^{103}$ \\
\hline 2019 & Quasi-2D & ITO/PEDOT:PSS/Pero/TPBi/LiF/AI & $487 /-$ & 11 & 9048 & - & $\sim 3$ & Ref. $^{98}$ \\
\hline 2019 & Quasi-2D & $\begin{array}{c}\text { ITO/NiOx:F6TCNNQ/PVK/Pero/TPBi/Cs } \\
\text { 2CO3/AI }\end{array}$ & $456 /-, 468 /-$ & 0.0150 .026 & - & - & - & Ref..$^{101}$ \\
\hline 2020 & Quasi-2D & ITO/PEDOT:PSS/Pero/TPBi/LiF/AI & $450 /-, 482 /-$ & $0.7,1.1$ & - & - & - & Ref. $^{96}$ \\
\hline 2020 & Quasi-2D & ITO/PEDOT:PSS/Pero/TyPmPB/LiF/AI & $494 /-$ & 2.7 & 465 & 4.3 & 3 & Ref. $^{93}$ \\
\hline 2020 & Quasi-2D & $\begin{array}{l}\text { ITO/PEDOT:PSS:PFI or POLY- } \\
\text { TPD/Pero/TPBi/LiF/AI }\end{array}$ & $489 / 18,479 / 18$ & $1.3,5.2$ & 5184,412 & - & $\sim 4$ & Ref. ${ }^{99}$ \\
\hline 2020 & Quasi-2D & ITO/PEDOT: PSS/Pero/TPBi/Ca/AI & $485 /-$ & 7.84 & 1130 & - & 4.3 & Ref. ${ }^{100}$ \\
\hline
\end{tabular}

methods are also useful in the precise control of the dimension distribution of quasi-2D perovskites, which exhibit potential in fabricating PeLEDs with emission spectra in the ranges of deep-blue and ultraviolet.
Features and outlook for device structure

In addition to perovskite films, the proper design of device structure can also improve the EL performance of blue PeLEDs. The strategies for the optimization of 
device structure of blue PeLEDs can be summarized in three categories: 1) assembling the carrier transport materials properly, 2) adopting multi-layer HTL materials, and 3) utilizing functional materials to modify the CTL/EML interface. All these strategies aim to improve the device performance from several aspects: 1) Improving the charge carrier concentration in the perovskite film. This can be achieved by either increasing the charge mobility of HTM/ETM or minimizing the energy offset between the CTLs and EML. 2) Diminishing the nonradiative recombination at the interface. Some commonly used HTL materials like PEDOT:PSS and $\mathrm{NiO}_{x}$ have features of exciton quenching, which can degrade the EL performance of blue PeLEDs. Strategies such as adopting multiple transport layers and inserting functional materials should be applied to modify these imperfect HTL materials. 3) Improving the morphology of perovskite films. As the morphology of perovskite films is related to the properties of underlying substrates, such as hydrophilicity/hydrophobicity, ionicity, and surface roughness. The chemical reactivity of underlying materials with the perovskite precursor should also be considered as perovskites can suffer a phase transition with reaction of the underlying substrates, which has previously been reported for red and green PeLEDs ${ }^{23,111}$. In fact, the synergistic optimization of both the perovskite films and device structure should be applied to improve the EL performance of blue PeLEDs to the maximum extent. As for device stability, even though the operating lifetime is a remaining challenge to be solved, strategies including surface passivation, additive engineering, composition modification, interface modification, and all-inorganic device structure that have been used to prolong the operating lifetimes of red and green PeLEDs can also be employed to promote the operating lifetime of blue PeLEDs.

\section{References}

1. Wehrenfennig C, Eperon GE, Johnston MB, Snaith HJ, Herz LM. High charge carrier mobilities and lifetimes in organolead trihalide perovskites. Adv Mater 26, 1584-1589 (2014).

2. Stranks SD, Eperon GE, Grancini G, Menelaou C, Alcocer MJP et al. Electron-hole diffusion lengths exceeding 1 micrometer in an organometal trihalide perovskite absorber. Science 342, 341-344 (2013).

3. Xiao ZG, Dong QF, Bi C, Shao YC, Yuan YB et al. Solvent annealing of perovskite-induced crystal growth for photovoltaicdevice efficiency enhancement. Adv Mater 26, 6503 -6509 (2014).

4. Peng SM, Wang SS, Zhao DD, Li XJ, Liang $C$ et al. Pure bromide-based perovskite nanoplatelets for blue light-emitting diodes. Small Methods 3, 1900196 (2019).

5. Luo DY, Su R, Zhang W, Gong QH, Zhu R. Minimizing non-radiative recombination losses in perovskite solar cells. Nat Rev Mater 5, 44-60 (2020).

6. Tian W, Zhou HP, Li L. Hybrid organic-inorganic perovskite photodetectors. Small 13, 1702107 (2017).

7. Veldhuis SA, Boix PP, Yantara N, Li MJ, Sum TC et al. Perovskite materials for light-emitting diodes and lasers. Adv Mater 28, 6804-6834 (2016).

8. Sahli F, Werner J, Kamino BA, Bräuninger M, Monnard R et al. Fully textured monolithic perovskite/silicon tandem solar cells with $25.2 \%$ power conversion efficiency. Nat Mater $\mathbf{1 7}$, 820-826 (2018).

9. Kong $\mathrm{CY}$, Lin $\mathrm{CH}$, Lin $\mathrm{CH}$, Li TY, Chen SWH et al. Highly efficient and stable white light-emitting diodes using perovskite quantum dot paper. Adv Sci 6, 1970143 (2019).

10. Li ZT, Song CJ, Li JS, Liang GW, Rao LS et al. Highly efficient and water-stable lead halide perovskite quantum dots using superhydrophobic aerogel inorganic matrix for white light-emitting diodes. Adv Mater Technol 5, 1900941 (2020).

11. Chen $P$, Xiong $Z Y, W u X Y$, Shao $M$, Meng $Y$ et al. Nearly $100 \%$ efficiency enhancement of $\mathrm{CH}_{3} \mathrm{NH}_{3} \mathrm{PbBr}_{3}$ perovskite light-emitting diodes by utilizing plasmonic au nanoparticles. $J$ Phys Chem Lett 8, 3961-3969 (2017).

12. Li ZT, Cao K, Li JS, Du XW, Tang $Y$ et al. Modification of interface between PEDOT:PSS and perovskite film inserting an ultrathin LiF layer for enhancing efficiency of perovskite lightemitting diodes. Org Electron 81, 105675 (2020).

13. Lu M, Zhang Y, Wang SX, Guo J, Yu WW et al. Metal halide perovskite light-emitting devices: promising technology for next-generation displays. Adv Funct Mater 29, 1902008 (2019).

14. Tan ZK, Moghaddam RS, Lai ML, Docampo P, Higler R et al. Bright light-emitting diodes based on organometal halide perovskite. Nat Nanotechnol 9, 687-692 (2014).

15. Li GR, Tan ZK, Di DW, Lai ML, Jiang $L$ et al. Efficient lightemitting diodes based on nanocrystalline perovskite in a dielectric polymer matrix. Nano Lett 15, 2640-2644 (2015).

16. Li JQ, Bade SGR, Shan X, Yu ZB. Single-layer light-emitting diodes using organometal halide perovskite/poly(ethylene oxide) composite thin films. Adv Mater 27, 5196-5202 (2015).

17. Yuan MJ, Quan LN, Comin R, Walters G, Sabatini R et al. Perovskite energy funnels for efficient light-emitting diodes. Nat Nanotechnol 11, 872-877 (2016).

18. Zhang CX, Kuang DB, Wu WQ. A review of diverse halide perovskite morphologies for efficient optoelectronic applications. Small Methods 4, 1900662 (2020).

19. Wang JP, Wang NN, Jin YZ, Si JJ, Tan ZK et al. Interfacial control toward efficient and low-voltage perovskite light-emitting diodes. Adv Mater 27, 2311-2316 (2015).

20. Shi YF, Wu W, Dong H, Li GR, Xi K et al. A strategy for architecture design of crystalline perovskite light-emitting diodes with high performance. Adv Mater 30, e1800251 (2018).

21. Cho H, Jeong $\mathrm{SH}$, Park $\mathrm{MH}$, Kim $\mathrm{YH}$, Wolf $\mathrm{C}$ et al. Overcoming the electroluminescence efficiency limitations of perovskite light-emitting diodes. Science 350, 1222-1225 (2015).

22. Lin KB, Xing J, Quan LN, de Arquer FPG, Gong XW et al. Perovskite light-emitting diodes with external quantum efficiency exceeding 20 per cent. Nature 562, 245-248 (2018). 
23. Cao $\mathrm{Y}$, Wang NN, Tian H, Guo JS, Wei YQ et al. Perovskite light-emitting diodes based on spontaneously formed submicrometre-scale structures. Nature 562, 249-253 (2018).

24. Xu WD, Hu Q, Bai S, Bao CX, Miao YF et al. Rational molecular passivation for high-performance perovskite light-emitting diodes. Nat Photonics 13, 418-424 (2019).

25. Li ZT, Song CJ, Qiu ZY, Li JS, Cao K et al. Study on the thermal and optical performance of quantum dot white lightemitting diodes using metal-based inverted packaging structure. IEEE Trans Electron Devices 66, 3020-3027 (2019).

26. Li CHA, Zhou ZC, Vashishtha P, Halpert JE. The future is blue (LEDs): why chemistry is the key to perovskite displays. Chem Mater 31, 6003-6032 (2019).

27. Zhou XJ, Tian PF, Sher CW, Wu J, Liu HZ et al. Growth, transfer printing and colour conversion techniques towards full-colour micro-LED display. Prog Quantum Electron 71, 100263 (2020).

28. Liu ZJ, Lin CH, Hyun BR, Sher CW, Lv ZJ et al. Micro-lightemitting diodes with quantum dots in display technology. Light Sci App/ 9, 83 (2020).

29. Li JS, Tang Y, Li ZT, Ding XR, Yu BH et al. Largely enhancing luminous efficacy, color-conversion efficiency, and stability for quantum-dot white LEDs using the two-dimensional hexagonal pore structure of sba-15 mesoporous particles. ACS Appl Mater Interfaces 11, 18808-18816 (2019).

30. Chen SWH, Huang YM, Singh KJ, Hsu YC, Liou FJ et al. Fullcolor micro-LED display with high color stability using semipolar (20-21) InGaN LEDs and quantum-dot photoresist. Photonics Res 8, 630-636 (2020).

31. Li ZT, Cao K, Li JS, Tang Y, Xu L et al. Investigation of lightextraction mechanisms of multiscale patterned arrays with rough morphology for gan-based thin-film LEDs. IEEE Access 7, 73890-73898 (2019).

32. Fang T, Zhang FJ, Yuan SC, Zeng HB, Song JZ. Recent advances and prospects toward blue perovskite materials and light-emitting diodes. InfoMat 1, 211-233 (2019).

33. Protesescu L, Yakunin S, Bodnarchuk MI, Krieg F, Caputo R et al. Nanocrystals of cesium lead halide perovskites $\left(\mathrm{CsPbX}_{3}\right.$, $\mathrm{X}=\mathrm{Cl}, \mathrm{Br}$, and $\mathrm{I}$ ): novel optoelectronic materials showing bright emission with wide color gamut. Nano Lett 15, 3692-3696 (2015).

34. Schulz P, Edri E, Kirmayer S, Hodes G, Cahen D et al. Interface energetics in organo-metal halide perovskite-based photovoltaic cells. Energy Environ Sci 7, 1377-1381 (2014).

35. Kumawat NK, Dey A, Kumar A, Gopinathan SP, Narasimhan $\mathrm{KL}$ et al. Band gap tuning of $\mathrm{CH}_{3} \mathrm{NH}_{3} \mathrm{~Pb}\left(\mathrm{Br}_{1-x} \mathrm{Cl}_{x}\right)_{3}$ hybrid perovskite for blue electroluminescence. ACS Appl Mater Interfaces 7, 13119-13124 (2015).

36. Sadhanala A, Ahmad S, Zhao BD, Giesbrecht N, Pearce PM et al. Blue-green color tunable solution processable organolead chloride-bromide mixed halide perovskites for optoelectronic applications. Nano Lett 15, 6095-6101 (2015).

37. Wang ZB, Cheng T, Wang FZ, Dai SY, Tan ZA. Morphology engineering for high-performance and multicolored perovskite light-emitting diodes with simple device structures. Small 12, 4412-4420 (2016).

38. Kim HP, Kim J, Kim BS, Kim HM, Kim J et al. High-efficiency, blue, green, and near-infrared light-emitting diodes based on triple cation perovskite. Adv Opt Mater 5, 1600920 (2017).

39. Wang HL, Zhao XF, Zhang BH, Xie ZY. Blue perovskite light- emitting diodes based on $\mathrm{RbX}$-doped polycrystalline $\mathrm{CsPbBr}_{3}$ perovskite films. J Mater Chem C 7, 5596-5603 (2019).

40. Yuan F, Ran CX, Zhang L, Dong H, Jiao B et al. A cocktail of multiple cations in inorganic halide perovskite toward efficient and highly stable blue light-emitting diodes. ACS Energy Lett 5, 1062-1069 (2020).

41. Gangishetty MK, Sanders SN, Congreve $\mathrm{DN}$. $\mathrm{Mn}^{2+}$ doping enhances the brightness, efficiency, and stability of bulk perovskite light-emitting diodes. ACS Photonics 6, $1111-1117$ (2019).

42. Du PP, Li JH, Wang L, Liu J, Li SR et al. Vacuum-deposited blue inorganic perovskite light-emitting diodes. ACS Appl Mater Interfaces 11, 47083-47090 (2019).

43. Song JZ, Li JH, Li XM, Xu LM, Dong YH et al. Quantum dot light-emitting diodes based on inorganic perovskite cesium lead halides $\left(\mathrm{CsPbX}_{3}\right)$. Adv Mater 27, 7162-7167 (2015).

44. Pan J, Quan LN, Zhao YB, Peng W, Murali B et al. Highly efficient perovskite-quantum-dot light-emitting diodes by surface engineering. Adv Mater 28, 8718-8725 (2016).

45. Yang F, Chen HT, Zhang R, Liu XK, Zhang WZ et al. Efficient and spectrally stable blue perovskite light-emitting diodes based on potassium passivated nanocrystals. Adv Funct Mater 30, 1908760 (2020).

46. Yao JS, Wang L, Wang KH, Yin YC, Yang JN et al. Calciumtributylphosphine oxide passivation enables the efficiency of pure-blue perovskite light-emitting diode up to $3.3 \%$. Sci Bull 65, 1150-1153 (2020).

47. Dong YT, Wang YK, Yuan FL, Johnston A, Liu Y et al. Bipolarshell resurfacing for blue LEDs based on strongly confined perovskite quantum dots. Nat Nanotechnol 15, $668-674$ (2020).

48. Li GR, Rivarola FWR, Davis NJLK, Bai S, Jellicoe TC et al. Highly efficient perovskite nanocrystal light-emitting diodes enabled by a universal crosslinking method. Adv Mater 28, 3528-3534 (2016).

49. Ochsenbein ST, Krieg F, Shynkarenko Y, Raino G, Kovalenko MV. Engineering color-stable blue light-emitting diodes with lead halide perovskite nanocrystals. ACS Appl Mater Interfaces 11, 21655-21660 (2019).

50. Deng $W, X u X Z$, Zhang XJ, Zhang YD, Jin XC et al. Organometal halide perovskite quantum dot light-emitting diodes. Adv Funct Mater 26, 4797-4802 (2016).

51. Yao EP, Yang ZL, Meng L, Sun PY, Dong SQ et al. Highbrightness blue and white LEDs based on inorganic perovskite nanocrystals and their composites. Adv Mater 29, 1606859 (2017).

52. Li XM, Zhang K, Li JH, Chen J, Wu Y et al. Heterogeneous nucleation toward polar-solvent-free, fast, and one-pot synthesis of highly uniform perovskite quantum dots for wider color gamut display. Adv Mater Interfaces 5, 1800010 (2018).

53. Shin YS, Yoon YJ, Lee KT, Jeong J, Park SY et al. Vivid and fully saturated blue light-emitting diodes based on ligand-modified halide perovskite nanocrystals. ACS Appl Mater Interfaces 11, 23401-23409 (2019).

54. Shynkarenko Y, Bodnarchuk MI, Bernasconi C, Berezovska Y, Verteletskyi $V$ et al. Direct synthesis of quaternary alkylammonium-capped perovskite nanocrystals for efficient blue and green light-emitting diodes. ACS Energy Lett 4, 2703-2711 (2019).

55. Ye FH, Zhang HJ, Wang $P$, Cai JL, Wang $L$ et al. Spectral 
tuning of efficient $\mathrm{CsPbBr}_{x} \mathrm{Cl}_{3-x}$ blue light-emitting diodes via halogen exchange triggered by benzenesulfonates. Chem Mater 32, 3211-3218 (2020).

56. Todorovic P, Ma DX, Chen B, Quintero-Bermudez R, Saidaminov $\mathrm{Ml}$ et al. Spectrally tunable and stable electroluminescence enabled by rubidium doping of $\mathrm{CsPbBr}_{3}$ nanocrystals. Adv Opt Mater 7, 1901440 (2019).

57. Liu M, Zhong GH, Yin YM, Miao JS, Li K et al. Aluminumdoped cesium lead bromide perovskite nanocrystals with stable blue photoluminescence used for display backlight. Adv Sci 4, 1700335 (2017).

58. Yong ZJ, Guo SQ, Ma JP, Zhang JY, Li ZY et al. Doping-enhanced short-range order of perovskite nanocrystals for nearunity violet luminescence quantum yield. J Am Chem Soc 140, 9942-9951 (2018).

59. Ahmed GH, El-Demellawi JK, Yin J, Pan J, Velusamy DB et al. Giant photoluminescence enhancement in $\mathrm{CsPbCl}_{3}$ perovskite nanocrystals by simultaneous dual-surface passivation. ACS Energy Lett 3, 2301-2307 (2018).

60. Mondal N, De A, Samanta A. Achieving near-unity photoluminescence efficiency for blue-violet-emitting perovskite nanocrystals. ACS Energy Lett 4, 32-39 (2019).

61. Zhai Y, Bai X, Pan GC, Zhu JY, Shao H et al. Effective blue-violet photoluminescence through lanthanum and fluorine ions Co-doping for $\mathrm{CsPbCl}_{3}$ perovskite quantum dots. Nanoscale 11, 2484-2491 (2019).

62. Chen JK, Ma JP, Guo SQ, Chen YM, Zhao Q et al. High-efficiency violet-emitting all-inorganic perovskite nanocrystals enabled by alkaline-earth metal passivation. Chem Mater 31, 3974-3983 (2019).

63. Meng FY, Liu XY, Cai XY, Gong ZF, Li BB et al. Incorporation of rubidium cations into blue perovskite quantum dot light-emitting diodes via FaBr-modified multi-cation hot-injection method. Nanoscale 11, 1295-1303 (2019).

64. Hou SC, Gangishetty MK, Quan QM, Congreve DN. Efficient blue and white perovskite light-emitting diodes via manganese doping. Joule 2, 2421-2433 (2018).

65. Pan GC, Bai X, Xu W, Chen $X$, Zhai $Y$ et al. Bright blue light emission of $\mathrm{Ni}^{2+}$ ion-doped $\mathrm{CsPbCl}_{x} \mathrm{Br}_{3-x}$ perovskite quantum dots enabling efficient light-emitting devices. ACS Appl Mater Interfaces 12, 14195-14202 (2020).

66. Shi SS, Wang Y, Zeng SY, Cui Y, Xiao Y. Surface regulation of $\mathrm{CsPbBr}_{3}$ quantum dots for standard blue-emission with boosted plqy. Adv Opt Mater 8, 2000167 (2020).

67. Shao H, Zhai Y, Wu XF, Xu W, Xu L et al. High brightness blue light-emitting diodes based on $\mathrm{CsPb}(\mathrm{Cl} / \mathrm{Br})_{3}$ perovskite qds with phenethylammonium chloride passivation. Nanoscale 12, 11728-11734 (2020).

68. Zheng XP, Yuan S, Liu JK, Yin J, Yuan FL et al. Chlorine vacancy passivation in mixed halide perovskite quantum dots by organic pseudohalides enables efficient rec. 2020 blue lightemitting diodes. ACS Energy Lett 5, 793-798 (2020).

69. Chiba T, Ishikawa S, Sato J, Takahashi Y, Ebe $\mathrm{H}$ et al. Blue perovskite nanocrystal light-emitting devices via the ligand exchange with adamantane diamine. Adv Opt Mater 8, 2000289 (2020).

70. Wang LT, Shi ZF, Ma ZZ, Yang DW, Zhang F et al. Colloidal synthesis of ternary copper halide nanocrystals for high-efficiency deep-blue light-emitting diodes with a half-lifetime above 100 h. Nano Lett 20, 3568-3576 (2020).
71. Levchuk I, Osvet A, Tang XF, Brandl M, Perea JD et al. Brightly luminescent and color-tunable formamidinium lead halide perovskite $\mathrm{FAPbX}_{3}(\mathrm{X}=\mathrm{Cl}, \mathrm{Br}, \mathrm{I})$ colloidal nanocrystals. Nano Lett 17, 2765-2770 (2017).

72. Bekenstein Y, Koscher BA, Eaton SW, Yang PD, Alivisatos AP. Highly luminescent colloidal nanoplates of perovskite cesium lead halide and their oriented assemblies. J Am Chem Soc 137, 16008-16011 (2015).

73. Akkerman QA, Motti SG, Kandada ARS, Mosconi E, D'Innocenzo $\mathrm{V}$ et al. Solution synthesis approach to colloidal cesium lead halide perovskite nanoplatelets with monolayer-level thickness control. J Am Chem Soc 138, 1010-1016 (2016).

74. Kumar S, Jagielski J, Yakunin S, Rice P, Chiu YC et al. Efficient blue electroluminescence using quantum-confined twodimensional perovskites. ACS Nano 10, 9720-9729 (2016).

75. Zhang CY, Wan Q, Wang B, Zheng WL, Liu MM et al. Surface ligand engineering toward brightly luminescent and stable cesium lead halide perovskite nanoplatelets for efficient blue-lightemitting diodes. J Phys Chem C 123, 26161-26169 (2019).

76. Bohn BJ, Tong $\mathrm{Y}$, Gramlich $\mathrm{M}$, Lai $\mathrm{ML}$, Döblinger $\mathrm{M}$ et al. Boosting tunable blue luminescence of halide perovskite nanoplatelets through postsynthetic surface trap repair. Nano Lett 18, 5231-5238 (2018).

77. Yang D, Zou YT, Li PL, Liu QP, Wu LZ et al. Large-scale synthesis of ultrathin cesium lead bromide perovskite nanoplates with precisely tunable dimensions and their application in blue light-emitting diodes. Nano Energy 47, 235-242 (2018).

78. $\mathrm{Wu} Y$, Wei CT, Li XM, Li YL, Qiu SC et al. In situ passivation of $\mathrm{PbBr}_{6}{ }^{4-}$ octahedra toward blue luminescent $\mathrm{CsPbBr}_{3}$ nanoplatelets with near $100 \%$ absolute quantum yield. ACS Energy Lett 3, 2030-2037 (2018).

79. Peng SM, Wen ZL, Ye TK, Xiao XT, Wang KY et al. Effective surface ligand-concentration tuning of deep-blue luminescent $\mathrm{FAPbBr}_{3}$ nanoplatelets with enhanced stability and charge transport. ACS Appl Mater Interfaces 12, 31863-31874 (2020).

80. Hu HW, Salim T, Chen BB, Lam YM. Molecularly engineered organic-inorganic hybrid perovskite with multiple quantum well structure for multicolored light-emitting diodes. Sci Rep 6, 33546 (2016).

81. Congreve DN, Weidman MC, Seitz M, Paritmongkol W, Dahod NS et al. Tunable light-emitting diodes utilizing quantum-confined layered perovskite emitters. ACS Photonics 4, 476-481 (2017).

82. Cheng L, Cao Y, Ge R, Wei YQ, Wang NN et al. Sky-blue perovskite light-emitting diodes based on quasi-two-dimensional layered perovskites. Chin Chem Lett 28, 29-31 (2017).

83. Chen ZM, Zhang CY, Jiang XF, Liu MY, Xia RX et al. High-performance color-tunable perovskite light emitting devices through structural modulation from bulk to layered film. Adv Mater 29, 1603157 (2017).

84. Wang Q, Ren J, Peng XF, Ji XX, Yang XH. Efficient sky-blue perovskite light-emitting devices based on ethylammonium bromide induced layered perovskites. ACS Appl Mater Interfaces 9, 29901-29906 (2017).

85. Vashishtha $\mathrm{P}, \mathrm{Ng} \mathrm{M}$, Shivarudraiah SB, Halpert JE. High efficiency blue and green light-emitting diodes using ruddlesdenpopper inorganic mixed halide perovskites with butylammonium interlayers. Chem Mater 31, 83-89 (2019).

86. Wang $\mathrm{KH}$, Peng YD, Ge J, Jiang SL, Zhu BS et al. Efficient and color-tunable quasi-2D $\mathrm{CsPbBr}_{x} \mathrm{C}_{3-x}$ perovskite blue light- 
emitting diodes. ACS Photonics 6, 667-676 (2019).

87. Li ZC, Chen ZM, Yang YC, Xue QF, Yip HL et al. Modulation of recombination zone position for quasi-two-dimensional blue perovskite light-emitting diodes with efficiency exceeding $5 \%$. Nat Commun 10, 1027 (2019).

88. Zhang FJ, Cai B, Song JZ, Han BN, Zhang BS et al. Efficient blue perovskite light-emitting diodes boosted by $2 \mathrm{D} / 3 \mathrm{D}$ energy cascade channels. Adv Funct Mater 30, 2001732 (2020).

89. Jiang YZ, Qin CC, Cui MH, He TW, Liu KK et al. Spectra stable blue perovskite light-emitting diodes. Nat Commun 10, 1868 (2019).

90. Liu Y, Cui JY, Du K, Tian H, He ZF et al. Efficient blue lightemitting diodes based on quantum-confined bromide perovskite nanostructures. Nat Photonics 13, 760-764 (2019).

91. Xing J, Zhao YB, Askerka M, Quan LN, Gong XW et al. Colorstable highly luminescent sky-blue perovskite light-emitting diodes. Nat Commun 9, 3541 (2018).

92. Yuan S, Wang ZK, Xiao LX, Zhang CF, Yang SY et al. Optimization of low-dimensional components of quasi-2D perovskite films for deep-blue light-emitting diodes. Adv Mater 31, 1904319 (2019).

93. He LH, Xiao ZW, Yang XL, Wu YT, Lian YJ et al. Green and sky blue perovskite light-emitting devices with a diamine additive. J Mater Sci 55, 7691-7701 (2020).

94. Liang D, Peng YL, Fu YP, Shearer MJ, Zhang JJ et al. Colorpure violet-light-emitting diodes based on layered lead halide perovskite nanoplates. ACS Nano 10, 6897-6904 (2016).

95. Deng W, Jin XC, Lv Y, Zhang XJ, Zhang XH et al. 2D Ruddlesden-popper perovskite nanoplate based deep-blue light-emitting diodes for light communication. Adv Funct Mater 29, 1903861 (2019).

96. Chen H, Lin J, Kang J, Kong Q, Lu D et al. Structural and spectral dynamics of single-crystalline ruddlesden-popper phase halide perovskite blue light-emitting diodes. Sci Adv 6 , eaay4045 (2020).

97. Tan ZF, Luo JJ, Yang LB, Li X, Deng ZY et al. Spectrally stable ultra-pure blue perovskite light-emitting diodes boosted by square-wave alternating voltage. Adv Opt Mater 8, 1901094 (2020).

98. Wang Q, Wang XM, Yang Z, Zhou NH, Deng YH et al. Efficient sky-blue perovskite light-emitting diodes via photoluminescence enhancement. Nat Commun 10, 5633 (2019).

99. Ma DX, Todorović P, Meshkat S, Saidaminov MI, Wang YK et al. Chloride insertion-immobilization enables bright, narrowband, and stable blue-emitting perovskite diodes. J Am Chem Soc 142, 5126-5134 (2020).

100. Wang FZ, Wang ZY, Sun WD, Wang ZB, Bai YM et al. High performance quasi-2D perovskite sky-blue light-emitting diodes using a dual-ligand strategy. Small 16, e2002940 (2020).

101. Leung TL, Tam HW, Liu FZ, Lin JY, Ng AMC et al. Mixed spacer cation stabilization of blue-emitting $n=2$ ruddlesden- popper organic-inorganic halide perovskite films. Adv Opt Mater 8, 1901679 (2020)

102. Zeng SY, Shi SS, Wang SR, Xiao Y. Mixed-ligand engineering of quasi-2D perovskites for efficient sky-blue light-emitting diodes. J Mater Chem C 8, 1319-1325 (2020).

103. Jin Y, Wang ZK, Yuan S, Wang Q, Qin CC et al. Synergistic effect of dual ligands on stable blue quasi-2D perovskite lightemitting diodes. Adv Funct Mater 30, 1908339 (2020).

104. Zou YT, Xu H, Li SY, Song T, Kuai L et al. Spectral-stable blue emission from moisture-treated low-dimensional lead bromidebased perovskite films. ACS Photonics 6, 1728-1735 (2019).

105. Yusoff ARBM, Gavim AEX, Macedo AG, da Silva WJ, Schneider FK et al. High-efficiency, solution-processable, multilayer triple cation perovskite light-emitting diodes with copper sulfide-gallium-tin oxide hole transport layer and aluminumzinc oxide-doped cesium electron injection layer. Mater Today Chem 10, 104-111 (2018).

106. Hoye RLZ, Lai ML, Anaya M, Tong Y, Galkowski K et al. Identifying and reducing interfacial losses to enhance colorpure electroluminescence in blue-emitting perovskite nanoplatelet light-emitting diodes. ACS Energy Lett 4, 1181-1188 (2019).

107. Gangishetty MK, Hou SC, Quan QM, Congreve DN. Reducing architecture limitations for efficient blue perovskite light-emitting diodes. Adv Mater 30, e1706226 (2018).

108. Ren ZW, Xiao XT, Ma RM, Lin H, Wang K et al. Hole transport bilayer structure for quasi-2D perovskite based blue light-emitting diodes with high brightness and good spectral stability. Adv Funct Mater 29, 1905339 (2019).

109. Shin YS, Yoon YJ, Heo J, Song S, Kim JW et al. Functionalized PFN-X (X $=\mathrm{Cl}, \mathrm{Br}$, or I) for balanced charge carriers of highly efficient blue light-emitting diodes. ACS Appl Mater Interfaces 12, 35740-35747 (2020).

110. Wang $\mathrm{HL}, \mathrm{Xu}$ YS, Wu J, Chen L, Yang $Q Q$ et al. Bright and color-stable blue-light-emitting diodes based on three-dimensional perovskite polycrystalline films via morphology and interface engineering. J Phys Chem Lett 11, 1411-1418 (2020).

111. Yuan ZC, Miao YF, Hu ZJ, Xu WD, Kuang CY et al. Unveiling the synergistic effect of precursor stoichiometry and interfacial reactions for perovskite light-emitting diodes. Nat Commun 10, 2818 (2019).

\section{Acknowledgements}

This work was supported by the National Natural Science Foundation of China (51775199, 51735004), Natural Science Foundation of Guangdong Province (2018B030306008) and the Fundamental Research Funds for the Central Universities. The authors would like to thank Professor Hao-Chung Kuo for his invitation and valuable comments on this review.

\section{Competing interests}

The authors declare no competing financial interests. 